\title{
Parametricity and Dependent Types
}

\author{
Jean-Philippe Bernardy Patrik Jansson \\ Chalmers University of Technology and \\ University of Gothenburg \\ \{bernardy,patrikj\}@chalmers.se
}

\author{
Ross Paterson \\ City University London \\ ross@soi.city.ac.uk
}

\begin{abstract}
Reynolds' abstraction theorem shows how a typing judgement in System F can be translated into a relational statement (in second order predicate logic) about inhabitants of the type. We obtain a similar result for a single lambda calculus (a pure type system), in which terms, types and their relations are expressed. Working within a single system dispenses with the need for an interpretation layer, allowing for an unusually simple presentation. While the unification puts some constraints on the type system (which we spell out), the result applies to many interesting cases, including dependently-typed ones.
\end{abstract}

Categories and Subject Descriptors $\quad$ F.3.3 [Logics and Meanings of Programs]: Studies of Program Constructs-Type Structure

General Terms Languages, Theory

Keywords Pure type system, Abstraction theorem, Free theorems

\section{Introduction}

Reynolds [1983] defined a relational interpretation of System F types, and showed that interpretations of a term of that type in related contexts yield related results. He was thus able to constrain interpretations of polymorphic types.

Wadler [1989] observed that if a type has no free variables, the relational interpretation can thus be viewed as a parametricity property satisfied by all terms of that type. Such properties have been used in a variety of situations. A few examples include:

program transformation The fold/build rule can be used to remove intermediate lists [Gill et al. 1993]. Its correctness can be proved using the parametricity condition derived from the type of the function build [Johann 2002].

testing The testing of a polymorphic function can often be reduced to testing a single monomorphic instance. Bernardy et al. [2010a] present a scheme for constructing such a monomorphic instance for which the correctness proof relies on parametricity.

automatic program inversion It is possible to write a function that inverts a polymorphic function given as input. The inversion process essentially relies on the parametric behaviour of the input function, and therefore its correctness relies on the corresponding parametricity condition [Voigtländer 2009a].

Permission to make digital or hard copies of all or part of this work for personal or classroom use is granted without fee provided that copies are not made or distributed for profit or commercial advantage and that copies bear this notice and the full citation on the first page. To copy otherwise, to republish, to post on servers or to redistribute to lists, requires prior specific permission and/or a fee.

ICFP'10, September 27-29, 2010, Baltimore, Maryland, USA.

Copyright (C) 2010 ACM 978-1-60558-794-3/10/09...\$10.00 generic programming In a certain style of generic programming, functions can be type-indexed. However, in some cases it is useful to show that functions behave uniformly for all types. Vytiniotis and Weirich [2009] use parametricity to show that certain casting functions are equivalent to the identity.

encoding of inductive types Via Church-encodings, inductive types can be encoded in pure System F. The proof of isomorphism relies on the parametricity condition. Hinze [2005] gives an illuminating example.

Parametricity in System $\mathrm{F}$ is useful enough that there has been much research to transport it to related calculi. Johann and Voigtländer [2005] have applied it to a system with explicit strictness; Vytiniotis and Weirich [2010] to $F_{\omega}$ extended with representation types; Takeuti [2004] sketches how it can be applied to the $\lambda$-cube, Neis et al. [2009] to a system with dynamic casting. In this paper, we apply Reynolds' idea to dependently-typed systems. In fact, we go one step further and generalize to a large class of pure type systems [Barendregt 1992].

By targeting pure type systems (PTSs), we aim to provide a framework which unifies previous descriptions of parametricity and forms a basis for future studies of parametricity in specific type systems. As a by-product, we get parametricity for dependentlytyped languages.

Our specific contributions are:

- A concise definition of the translation of types to relations (Definition 4), which yields parametricity propositions for PTSs.

- A formulation (and a proof) of the abstraction theorem for a useful class of PTSs (Theorem 1). A remarkable feature of the theorem is that the translation from types to relations and the translations from terms to proofs are unified.

- An extension of the translation to inductive definitions (Section 4). Our examples use a notation close to that of Agda [Norell 2007], for greater familiarity for users of dependentlytyped functional programming languages.

- A demonstration by example of how to derive free theorems for (and as) dependently-typed functions (sections 3.1 and 5). Two examples of functions that we tackle are:

generic catamorphism fold : $\left(\left(F\right.\right.$, map $\left._{F}\right):$ Functor $) \rightarrow$ $(A: \star) \rightarrow(F A \rightarrow A) \rightarrow \mu F \rightarrow A$, which is a generic catamorphism function defined within a dependently-typed language (see Section 5.2).

generic cast gcast : $(F: \star \rightarrow \star) \rightarrow(u t: U) \rightarrow$ Maybe $(F(E l u) \rightarrow F(E l t))$, which comes from a modelling of representation types with universes (see Section 5.3).

In both cases, the derived parametricity condition yields useful properties to reason about the correctness of the function. 


\section{Pure type systems}

In this section we briefly review the notion of PTS as described by Barendregt [1992, sec. 5.2], and the basic intuitions behind it. We introduce our notation along the way, as well as our running example type system.

Definition 1 (Syntax of terms). A PTS is a type system over a $\lambda$ calculus with the following syntax:

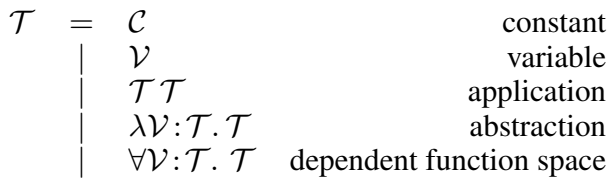

We often write $(x: \mathrm{A}) \rightarrow \mathrm{B}$ for $\forall x: \mathrm{A}$. $\mathrm{B}$, and sometimes just $\mathrm{A} \rightarrow \mathrm{B}$ when $x$ does not occur free in $\mathrm{B}$.

The typing judgement of a PTS is parametrized over a specification $S=(\mathcal{S}, \mathcal{A}, \mathcal{R})$, where $\mathcal{S} \subseteq \mathcal{C}, \mathcal{A} \subseteq \mathcal{C} \times \mathcal{S}$ and $\mathcal{R} \subseteq \mathcal{S} \times \mathcal{S} \times \mathcal{S}$. The set $\mathcal{S}$ specifies the sorts, $\mathcal{A}$ the axioms (an axiom $(c, s) \in \mathcal{A}$ is often written $c: s$ ), and $\mathcal{R}$ specifies the typing rules of the function space. A rule $\left(s_{1}, s_{2}, s_{2}\right)$ is often written $s_{1} \leadsto s_{2}$. The rules for typing judgements in a PTS are given in Figure 1.

An attractive feature of PTSs is that the syntax for types and values is unified. It is the type of a term that tells how to interpret it (as a value, type, kind, etc.).

the $\lambda$-cube Barendregt [1992] defined a family of calculi each with $\mathcal{S}=\{\star, \square\}, \mathcal{A}=\{\star: \square\}$ and $\mathcal{R}$ a selection of rules of the form $s_{1} \leadsto s_{2}$, for example:

- The (monomorphic) $\lambda$-calculus has $\mathcal{R}_{\lambda}=\{\star \sim \star\}$, corresponding to ordinary functions.

- System $\mathrm{F}$ has $\mathcal{R}_{\mathrm{F}}=\mathcal{R}_{\lambda} \cup\{\square \leadsto \star\}$, adding (impredicative) universal quantification over types.

- System $\mathrm{F} \omega$ has $\mathcal{R}_{\mathrm{F} \omega}=\mathcal{R}_{\mathrm{F}} \cup\{\square \sim \square\}$, adding type-level functions.

- The Calculus of Constructions (CC) has $\mathcal{R}_{\mathrm{CC}}=\mathcal{R}_{\mathrm{F} \omega} \cup\{\star \leadsto$ $\square$ \}, adding dependent types.

Here $\star$ and $\square$ are conventionally called the sorts of types and kinds respectively.

Notice that $\mathrm{F}$ is a subsystem of $\mathrm{F} \omega$, which is itself a subsystem of CC. (We say that $S_{1}=\left(\mathcal{S}_{1}, \mathcal{A}_{1}, \mathcal{R}_{1}\right)$ is a subsystem of $S_{2}=$ $\left(\mathcal{S}_{2}, \mathcal{A}_{2}, \mathcal{R}_{2}\right)$ when $\mathcal{S}_{1} \subseteq \mathcal{S}_{2}, \mathcal{A}_{1} \subseteq \mathcal{A}_{2}$ and $\mathcal{R}_{1} \subseteq \mathcal{R}_{2}$.) In fact, the $\lambda$-cube is so named because the lattice of the subsystem relation between all the systems forms a cube, with $\mathrm{CC}$ at the top.

sort hierarchies Difficulties with impredicativity ${ }^{1}$ have led to the development of type systems with an infinite hierarchy of sorts. The "pure" part of such a system can be captured in the following PTS, which we name $I_{\omega}$.

Definition $2\left(I_{\omega}\right) . I_{\omega}$ is a PTS with this specification:

- $\mathcal{S}=\left\{\star_{i} \mid i \in \mathbb{N}\right\}$

- $\mathcal{A}=\left\{\star_{i}: \star_{i+1} \mid i \in \mathbb{N}\right\}$

- $\mathcal{R}=\left\{\left(\star_{i}, \star_{j}, \star_{\max (i, j)}\right) \mid i, j \in \mathbb{N}\right\}$

Compared to the monomorphic $\lambda$-calculus, $\star$ has been expanded into the infinite hierarchy $\star_{0}, \star_{1}, \ldots$ In $I_{\omega}$, the sort $\star_{0}$ (abbreviated $\star$ ) is called the sort of types. Type constructors, or type-level functions have type $\star \rightarrow \star$. The set of types $(\star)$, the set of type constructors $(\star \rightarrow \star)$ and similar have type $\star_{1}$ (the sort of kinds). Terms like $\star_{1}$ and $\star \rightarrow \star_{1}$ have type $\star_{2}$, and so on.

Impredicativity can in fact coexist with an infinite hierarchy of sorts, as Coquand [1986] has shown. For example, in the Gen-

\footnotetext{
${ }^{1}$ It is inconsistent with strong sums [Coquand 1986].
}

$$
\begin{aligned}
& \text { axiom } \frac{}{\vdash c: s} c: s \in \mathcal{A} \\
& \operatorname{start} \frac{\Gamma \vdash \mathrm{A}: s}{\Gamma, x: \mathrm{A} \vdash x: \mathrm{A}} \\
& \text { weakening } \frac{\Gamma \vdash \mathrm{A}: \mathrm{B} \quad \Gamma \vdash \mathrm{C}: s}{\Gamma, x: \mathrm{C} \vdash \mathrm{A}: \mathrm{B}} \\
& \text { product } \frac{\Gamma \vdash \mathrm{A}: s_{1} \quad \Gamma, x: \mathrm{A} \vdash \mathrm{B}: s_{2}}{\Gamma \vdash(\forall x: \mathrm{A} . \mathrm{B}): s_{3}}\left(s_{1}, s_{2}, s_{3}\right) \in \mathcal{R} \\
& \text { application } \frac{\Gamma \vdash \mathrm{F}:(\forall x: \mathrm{A} . \mathrm{B}) \quad \Gamma \vdash \mathrm{a}: \mathrm{A}}{\Gamma \vdash \mathrm{Fa}: \mathrm{B}[x \mapsto \mathrm{a}]} \\
& \text { abstraction } \frac{\Gamma, x: \mathrm{A} \vdash \mathrm{b}: \mathrm{B} \quad \Gamma \vdash(\forall x: \mathrm{A} . \mathrm{B}): s}{\Gamma \vdash(\lambda x: \mathrm{A} . \mathrm{b}):(\forall x: \mathrm{A} . \mathrm{B})} \\
& \text { conversion } \frac{\Gamma \vdash \mathrm{A}: \mathrm{B} \quad \Gamma \vdash \mathrm{B}^{\prime}: s \quad \mathrm{~B}={ }_{\beta} \mathrm{B}^{\prime}}{\Gamma \vdash \mathrm{A}: \mathrm{B}^{\prime}}
\end{aligned}
$$

Figure 1. Typing judgements of the $\operatorname{PTS}(\mathcal{S}, \mathcal{A}, \mathcal{R})$

eralized Calculus of Constructions $\left(\mathrm{CC}_{\omega}\right)$ of Miquel [2001], impredicativity exists for the sort $\star$ (conventionally called the sort of propositions), which lies at the bottom of the hierarchy.

Definition $3\left(\mathrm{CC}_{\omega}\right) \cdot \mathrm{CC}_{\omega}$ is a PTS with this specification:

- $\mathcal{S}=\{\star\} \cup\left\{\square_{i} \mid i \in \mathbb{N}\right\}$

- $\mathcal{A}=\left\{\star: \square_{0}\right\} \cup\left\{\square_{i}: \square_{i+1} \mid i \in \mathbb{N}\right\}$

- $\mathcal{R}=\left\{\star \leadsto \star, \star \leadsto \square_{i}, \square_{i} \leadsto \star \mid i \in \mathbb{N}\right\} \cup$

$$
\left\{\left(\square_{i}, \square_{j}, \square_{\max (i, j)}\right) \mid i, j \in \mathbb{N}\right\}
$$

Both CC and $I_{\omega}$ are subsystems of $\mathrm{CC}_{\omega}$, with $\star_{i}$ in $I_{\omega}$ corresponding to $\square_{i}$ in $\mathrm{CC}_{\omega}$. Because $\square$ in CC corresponds to $\square_{0}$ in $\mathrm{CC}_{\omega}$, we often abbreviate $\square_{0}$ as $\square$.

Many dependently-typed programming languages and proof assistants are based on variants of $I_{\omega}$ or $\mathrm{CC}_{\omega}$, often with the addition of inductive definitions [Dybjer 1994; Paulin-Mohring 1993]. Such tools include Agda [Norell 2007], Coq [The Coq development team 2010] and Epigram [McBride and McKinna 2004].

\subsection{PTS as logical system}

Another use for PTSs is as logical systems: types correspond to propositions and terms to proofs. This correspondence extends to all aspects of the systems and is widely known as the Curry-Howard isomorphism. The judgement $\vdash \mathrm{p}: \mathrm{P}$ means that $\mathrm{p}$ is a witness, or proof of the proposition $\mathrm{P}$.

In the logical system reading, an inhabited type corresponds to a tautology and dependent function types correspond to universal quantification. Predicates over a type $\mathrm{A}$ have type $\mathrm{A} \rightarrow s$, for some sort $s$ : a value satisfies the predicate whenever the returned type is inhabited. Similarly, binary relations between values of types $A_{1}$ and $\mathrm{A}_{2}$ have type $\mathrm{A}_{1} \rightarrow \mathrm{A}_{2} \rightarrow s$.

For this approach to be safe, it is important that the system be consistent: some types must be uninhabited, or equivalently each witness $\mathrm{p}$ must reduce to a normal form. This is the case for the systems used here.

In fact, in $I_{\omega}$ and similarly rich type systems, one may both represent programs and logical formulae about them. In the following sections, we make full use of this property: we encode programs and parametricity statements about them in the same type system. 


\section{Types to relations}

We start by defining the relational interpretation of a term, as a syntactic translation from terms to terms. As we see in Section 3.1, it is a generalization of the classical rules given by Reynolds [1983], extended to application and abstraction.

In this section, we assume that the only constants are sorts. We also assume for each sort $s$ another sort $\widetilde{s}$ of parametricity propositions about terms of type $s$. In our examples, we simply choose $\widetilde{s}=s$. We shall return to the general case in Section 6.2.

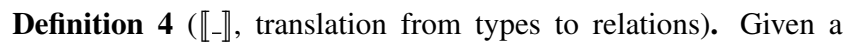
natural number $n$ (the arity of relations), we assume for each variable $x$, fresh variables $x_{1}, \ldots, x_{n}$ and $x_{R}$. We write $\overline{\mathrm{A}}$ for the $n$ terms $\mathrm{A}_{i}$, each obtained by replacing each free variable $x$ in $\mathrm{A}$ with $x_{i}$. Correspondingly, $\overline{x: \mathrm{A}}$ stands for $n$ bindings $\left(x_{i}: \mathrm{A}_{i}\right)$. We define a mapping $\llbracket-\rrbracket$ from $\mathcal{T}$ to $\mathcal{T}$ as follows:

$$
\begin{aligned}
\llbracket s \rrbracket & =\lambda \overline{x: s} \cdot \bar{x} \rightarrow \widetilde{s} \\
\llbracket x \rrbracket & =x_{R} \\
\llbracket \forall x: \mathrm{A} . \mathrm{B} \rrbracket & =\lambda \overline{f:(\forall x: \mathrm{A} . \mathrm{B})} \cdot \forall \overline{x: \mathrm{A}} \cdot \forall x_{R}: \llbracket \mathrm{A} \rrbracket \bar{x} \cdot \llbracket \mathrm{B} \rrbracket(\overline{f x}) \\
\llbracket \mathrm{F} \mathrm{a} \rrbracket & =\llbracket \mathrm{F} \rrbracket \overline{\mathrm{a} \llbracket \mathrm{a} \rrbracket} \\
\llbracket \lambda x: \mathrm{A} \cdot \mathrm{b} \rrbracket & =\lambda \overline{x: \mathrm{A}} \cdot \lambda x_{R}: \llbracket \mathrm{A} \rrbracket \bar{x} \cdot \llbracket \mathrm{b} \rrbracket
\end{aligned}
$$

Note that for each variable $x$ free in $A$, the translation $\llbracket A \rrbracket$ has free variables $x_{1}, \ldots, x_{n}$ and $x_{R}$. There is a corresponding replication of variables bound in contexts, which is made explicit in the following definition.

Definition 5 (translation of contexts).

$$
\llbracket \Gamma, x: \mathrm{A} \rrbracket=\llbracket \Gamma \rrbracket, \overline{x: \mathrm{A}}, x_{R}: \llbracket \mathrm{A} \rrbracket \bar{x}
$$

Note that each tuple $\bar{x}: \overline{\mathrm{A}}$ in the translated context must satisfy the relation $\llbracket \mathrm{A} \rrbracket$, as witnessed by $x_{R}$. Thus, one may interpret $\llbracket \Gamma \rrbracket$ as $n$ related environments.

In order for a PTS to be able to express both programs and parametricity propositions about them, it must satisfy certain closure conditions, for which we coin the term reflective:

Definition 6 (reflective). A PTS $(\mathcal{S}, \mathcal{A}, \mathcal{R})$ is reflective if

- for each sort $s \in \mathcal{S}$

- $\exists \widetilde{s} \in \mathcal{S}$

- $\exists s^{\prime} \in \mathcal{S}$ such that $s: s^{\prime} \in \mathcal{A}$

- for each axiom $s: s^{\prime} \in \mathcal{A}$

- $\widetilde{s}: \widetilde{s^{\prime}} \in \mathcal{A}$

- $s \leadsto \widetilde{s^{\prime}} \in \mathcal{R}$

- for each rule $\left(s_{1}, s_{2}, s_{3}\right) \in \mathcal{R}$

- $\left(\widetilde{s_{1}}, \widetilde{s_{2}}, \widetilde{s_{3}}\right) \in \mathcal{R}$

- $s_{1} \leadsto \widetilde{s_{3}} \in \mathcal{R}$

We can then state our main result:

Theorem 1 (abstraction). Given a reflective $\operatorname{PTS}(\mathcal{S}, \mathcal{A}, \mathcal{R})$, $\Gamma \vdash \mathrm{A}: \mathrm{B} \Longrightarrow \llbracket \Gamma \rrbracket \vdash \llbracket \mathrm{A} \rrbracket: \llbracket \mathrm{B} \rrbracket \overline{\mathrm{A}}$

Proof. By induction on the derivation. A brief sketch of the proof is given in appendix A.

The above theorem can be read in two ways. A direct reading is as a typing judgement about translated terms: if $A$ has type $B$, then $\llbracket A \rrbracket$ has type $\llbracket B \rrbracket \bar{A}$. The more fruitful reading is as an abstraction theorem for pure type systems: if $A$ has type $B$ in environment $\Gamma$, then $n$ interpretations $\overline{\mathrm{A}}$ in related environments $\llbracket \Gamma \rrbracket$ are related by $\llbracket \mathrm{B} \rrbracket$. Further, $\llbracket \mathrm{A} \rrbracket$ is a witness of this proposition within the type system. In particular, closed terms are related to themselves:

Corollary 2 (parametricity). $\vdash A: B \Longrightarrow \vdash \llbracket A \rrbracket: \llbracket B \rrbracket \bar{A}$ example systems Note that both $I_{\omega}$ and $\mathrm{CC}_{\omega}$ are reflective, with $\widetilde{s}=s$. Therefore we can write programs in these systems and derive valid statements about them, using 【-】, within the same PTS. We proceed to do so in the rest of the paper.

\subsection{Examples: the $\lambda$-cube}

In this section, we show that $\llbracket \_$specializes to the rules given by Reynolds [1983] to read a System F type as a relation. Having shown that our framework can explain parametricity theorems for System-F-style types, we move on to progressively higher-order constructs. In these examples, the binary version of parametricity is used (arity $n=2$ ). For examples using the unary version (arity $n=1$ ) see Section 5.3.

While the systems of the $\lambda$-cube are not reflective, they are embedded in $\mathrm{CC}_{\omega}$, which is. This means that our translation rules take System F types to terms in $\mathrm{CC}_{\omega}$ (instead of second order propositional logic). The possibility of using a different PTS for the logic is discussed in Section 6.3.

types to relations Note that, by definition,

$$
\llbracket \star \rrbracket T_{1} T_{2}=T_{1} \rightarrow T_{2} \rightarrow \star
$$

Assuming that types inhabit the sort $\star$, this means that types are translated to relations (as expected). Here we also use $\star$ on the right side as the sort of propositions $(\overleftarrow{\star}=\star$ ), but other choices are possible, as we shall discuss in Section 6.2.

function types Applying our translation to non-dependent function types, we get:

$$
\begin{aligned}
\llbracket \mathrm{A} \rightarrow \mathrm{B} \rrbracket: \llbracket \star \rrbracket( & \mathrm{A} \rightarrow \mathrm{B})(\mathrm{A} \rightarrow \mathrm{B}) \\
\llbracket \mathrm{A} \rightarrow \mathrm{B} \rrbracket f_{1} f_{2}= & \forall a_{1}: \mathrm{A} . \forall a_{2}: \mathrm{A} . \\
& \llbracket \mathrm{A} \rrbracket a_{1} a_{2} \rightarrow \llbracket \mathrm{B} \rrbracket\left(f_{1} a_{1}\right)\left(f_{2} a_{2}\right)
\end{aligned}
$$

That is, functions are related iff they take related arguments into related outputs.

type schemes System $\mathrm{F}$ includes universal quantification of the form $\forall A: \star$. B. Applying $\llbracket-\rrbracket$ to this type expression yields:

$$
\begin{aligned}
\llbracket \forall A: \star . \mathrm{B} \rrbracket: \llbracket \star \rrbracket(\forall A: \star . \mathrm{B})(\forall A: \star . \mathrm{B}) \\
\llbracket \forall A: \star . \mathrm{B} \rrbracket g_{1} g_{2}=\forall A_{1}: \star . \forall A_{2}: \star . \forall A_{R}: \llbracket \star \rrbracket A_{1} A_{2} . \\
\llbracket \mathrm{B} \rrbracket\left(g_{1} A_{1}\right)\left(g_{2} A_{2}\right)
\end{aligned}
$$

In words, polymorphic values are related iff instances at related types are related. Note that as $A$ may occur free in $\mathrm{B}$, the variables $A_{1}, A_{2}$ and $A_{R}$ may occur free in $\llbracket \mathrm{B} \rrbracket$.

type constructors $\quad$ With the addition of the rule $\square \sim \square$, one can construct terms of type $\star \rightarrow \star$, which are sometimes known as type constructors, type formers or type-level functions. As Voigtländer [2009b] remarks, extending Reynolds-style parametricity to support type constructors appears to be folklore. Such folklore can be precisely justified by our framework by applying 【-』 to obtain the relational counterpart of type constructors:

$$
\begin{aligned}
& \llbracket \star \rightarrow \star \rrbracket: \llbracket \square \rrbracket(\star \rightarrow \star)(\star \rightarrow \star) \\
& \llbracket \star \rightarrow \star \rrbracket F_{1} F_{2}=\forall A_{1}: \star . \forall A_{2}: \star . \\
& \llbracket \star \rrbracket A_{1} A_{2} \rightarrow \llbracket \star \rrbracket\left(F_{1} A_{1}\right)\left(F_{2} A_{2}\right)
\end{aligned}
$$

That is, a term of type $\llbracket \star \rightarrow \star \rrbracket F_{1} F_{2}$ is a (polymorphic) function converting a relation between any types $A_{1}$ and $A_{2}$ to a relation between $F_{1} A_{1}$ and $F_{2} A_{2}$, a relational action.

dependent functions In a system with the rule $\star \sim \square$, value variables may occur in dependent function types like $\forall x: \mathrm{A}$. B, which we translate as follows:

$$
\begin{gathered}
\llbracket \forall x: \text { A. B } \rrbracket: \llbracket \star \rrbracket(\forall x: \text { A. B })(\forall x: \text { A. B }) \\
\llbracket \forall x: \text { A. B } \rrbracket f_{1} f_{2}= \\
\forall x_{1}: \text { A. } \forall x_{2}: \text { A. } \forall x_{R}: \llbracket A \rrbracket x_{1} x_{2} . \\
\llbracket \text { B } \rrbracket\left(f_{1} x_{1}\right)\left(f_{2} x_{2}\right)
\end{gathered}
$$


proof terms We have used «-』 to turn types into relations, but we can also use it to turn terms into proofs of abstraction properties. As a simple example, the relation corresponding to the type $T=$ $\forall A: \star . A \rightarrow A$, namely

$$
\begin{aligned}
\llbracket T \rrbracket f_{1} f_{2}= & \forall A_{1}: \star . \forall A_{2}: \star . \forall A_{R}: \llbracket \star \rrbracket A_{1} A_{2} . \\
& \forall x_{1}: A_{1} . \forall x_{2}: A_{2} . \\
& A_{R} x_{1} x_{2} \rightarrow A_{R}\left(f_{1} A_{1} x_{1}\right)\left(f_{2} A_{2} x_{2}\right)
\end{aligned}
$$

states that functions of this type map related inputs to related outputs. From a term $i d=\lambda A: \star . \lambda x: A . x$ of this type, by Theorem 2 we obtain a term $\llbracket i d \rrbracket: \llbracket T \rrbracket i d i d$, that is, a proof of the abstraction property:

$$
\llbracket i d \rrbracket A_{1} A_{2} A_{R} x_{1} x_{2} x_{R}=x_{R}
$$

\section{Constants and data types}

While the above development assumes pure type systems with $\mathcal{C}=\mathcal{S}$, it is possible to add constants to the system and retain parametricity, as long as each constant is parametric. That is, for each new axiom $\vdash k: \mathrm{A}$ (where $k$ is an arbitrary constant and $\mathrm{A}$ an arbitrary term such that $\vdash \mathrm{A}: s$, not a mere sort) we require a term $\llbracket k \rrbracket$ such that the judgement $\vdash \llbracket k \rrbracket: \llbracket \mathrm{A} \rrbracket \bar{k}$ holds. (Additionally, $\beta$ conversion rules involving those constants must preserve types.)

One source of constants in many languages is data type definitions. In the rest of the this section we detail how to handle such definitions (in a system extending $I_{\omega}$ ).

\subsection{Inductive families}

Many languages permit data type declarations like those in Figure 2 . Dependently typed languages typically allow the return types of constructors to have different arguments, yielding inductive families [Dybjer 1994; Paulin-Mohring 1993] such as the family Vec, in which the type is indexed by the number of elements.

Data family declarations of sort $s$ ( $\star$ in the examples) have the typical form: ${ }^{2}$

$$
\begin{gathered}
\text { data } T(a: \mathrm{A}): \forall n: \text { N. } s \text { where } \\
c: \forall b: \text { B. }(\forall x: \text { X. Ta i }) \rightarrow T a \vee
\end{gathered}
$$

Arguments of the type constructor $T$ may be either parameters $a$, which scope over the constructors and are repeated at each recursive use of $T$, or indices $n$, which may vary between uses. Data constructors $c$ have non-recursive arguments $b$, whose types are otherwise unrestricted, and recursive arguments with types of a constrained form, which cannot be referred to in the other terms.

Such a declaration can be interpreted as a simultaneous declaration of formation and introduction constants

$$
\begin{aligned}
& T: \forall a: \text { A. } \forall n: \text { N. } s \\
& c: \forall a: \text { A. } \forall b: \text { B. }(\forall x: \text { X. } T a \mathrm{i}) \rightarrow T a \text { v }
\end{aligned}
$$

and also an eliminator to analyse values of that type:

$$
\begin{aligned}
\text { T-elim }: & \forall a: \text { A. } \\
& \forall P:(\forall n: \text { N. Tan } \rightarrow s) . \\
& \text { Case }_{c} \rightarrow \forall n: \text { N. } \forall t: \text { Tan. Pnt }
\end{aligned}
$$

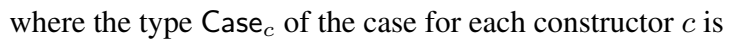

$$
\forall b: \text { B. } \forall u:(\forall x: \text { X. Tai }) .(\forall x: \text { X. } P \mathrm{i}(u x)) \rightarrow P \vee(c a b u)
$$

with beta-equivalences (one for each constructor $c$ ):

$$
\text { T-elim a P ev }(\text { cabu })=\text { ebu }(\lambda x: \mathbf{X} . T \text {-elim a Pei }(u x))
$$

We shall often use corresponding pattern matching definitions instead of these eliminators [Coquand 1992].

\footnotetext{
${ }^{2}$ We show only one of each element here, but the generalization to arbitrary numbers is straightforward.
}

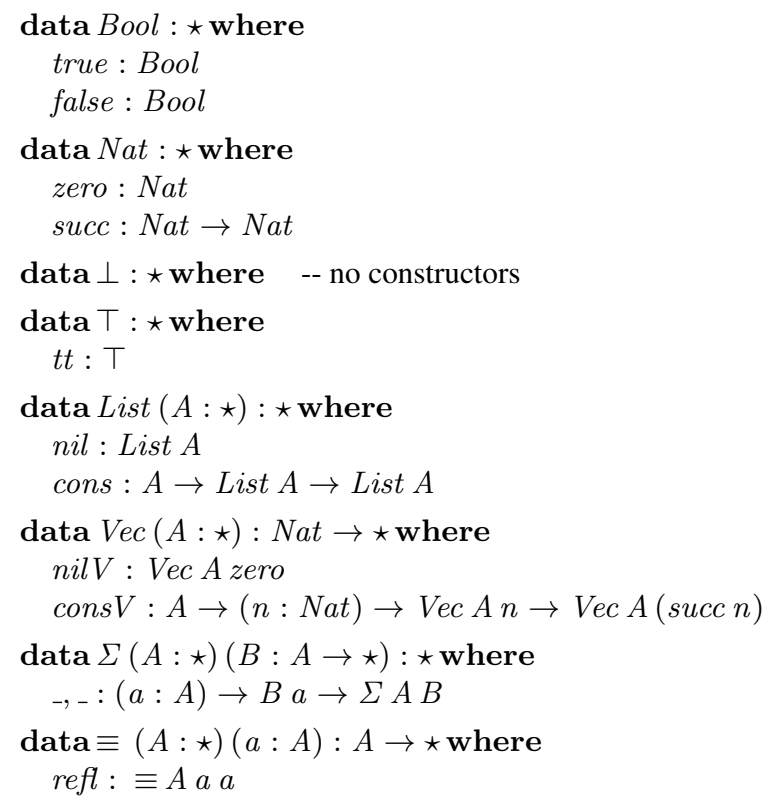

Figure 2. Example inductive families

For example, the definition of List in Figure 2 gives rise to the following constants:

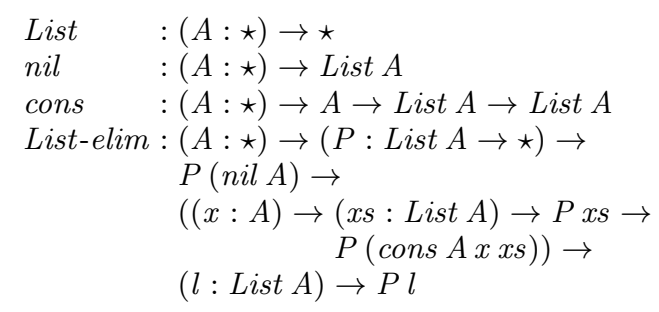

In the following sections, we consider two ways to define an abstraction proof $\llbracket k \rrbracket: \llbracket \tau \rrbracket \bar{k}$ for each constant $k: \tau$ introduced by the data definition.

\subsection{Deductive-style translation}

First, we define each proof as a term (using pattern matching to simplify the presentation). We begin with the translation of the equation for each constructor:

$$
\begin{array}{r}
\llbracket T \text {-elim a P e v } \overline{(c a b u)}\left(\llbracket c \rrbracket \bar{a} a_{R} \bar{b} b_{R} \bar{u} u_{R}\right)= \\
\llbracket e b u(\lambda x: X . T-e l i m \text { a } P \text { e } \mathrm{i}(u x)) \rrbracket
\end{array}
$$

To turn this into a pattern matching definition of T-elim, we need a suitable definition of $\llbracket c \rrbracket$, and similarly for the constructors in $\mathrm{v}$. The only arguments of $\llbracket c \rrbracket$ not already in scope are $b_{R}$ and $u_{R}$, so we package them as a dependent pair, because the type of $u_{R}$ may depend on that of $b_{R}$. Writing $(x: \mathrm{A}) \times \mathrm{B}$ for $\Sigma \mathrm{A}(\lambda x: \mathrm{A}$. B $)$, and elements of this type as $(a, b)$, omitting the arguments $A$ and $\lambda x: \mathrm{A} . \mathrm{B}$, we define $\mathrm{s}^{3}$

$$
\begin{aligned}
& \llbracket T \rrbracket: \llbracket \forall a: \mathrm{A} . \forall n: \mathrm{N} . s \rrbracket \bar{T} \\
& \llbracket T \rrbracket \bar{a} a_{R} \overline{\mathrm{v}} \llbracket \mathrm{v} \rrbracket\left(\overline{(c a b u)}=\left(b_{R}: \llbracket \mathrm{B} \rrbracket \bar{b}\right) \times \llbracket \forall x: \mathrm{X} . T a \mathrm{i} \rrbracket \bar{u}\right. \\
& \llbracket T \rrbracket \bar{a} a_{R} \bar{u} u_{R} \bar{t}=\perp \\
& \llbracket c \rrbracket: \llbracket \forall a: \mathrm{A} . \forall b: \mathrm{B} .(\forall x: \mathrm{X} . T a \mathrm{i}) \rightarrow T a \mathrm{v} \rrbracket \bar{c} \\
& \llbracket c \rrbracket \bar{a} a_{R} \bar{b} b_{R} \bar{u} u_{R}=\left(b_{R}, u_{R}\right)
\end{aligned}
$$

\footnotetext{
${ }^{3}$ The definition of $\llbracket T \rrbracket$ relies on the weak elimination constant to sort $\widetilde{s}^{\prime}$.
} 
and the translation of T-elim becomes

$$
\begin{aligned}
& \llbracket T \text {-elim a P e v } \rrbracket \overline{(c a b u)}\left(b_{R}, u_{R}\right)= \\
& \llbracket e b u(\lambda x: \text { X. T-elim a P e i }(u x)) \rrbracket
\end{aligned}
$$

Because $\llbracket T \rrbracket$ yields $\perp$ unless the constructors match, these clauses provide complete coverage.

The reader may have noted by now that the argument lists of the translated constants tend to be quite long. The use of the translated constants can be substantially simplified using implicit arguments (arguments which can be inferred from contextual knowledge). We avoid using them in this paper to explicitly show the underlying machinery, but the Agda library implementing the translation makes heavy use of implicit arguments for convenience.

Booleans To get an intuition of the meaning of the above translation scheme we proceed to apply it to a number of examples, starting with the data type for Booleans. We obtain:

$$
\begin{aligned}
& \llbracket \text { Bool } \rrbracket: \llbracket \star \rrbracket B o o l ~ B o o l \\
& \llbracket \text { Bool } \rrbracket \text { true true }=\top \\
& \llbracket \text { Bool false false }=\top \\
& \llbracket \text { Bool }{ }_{-} \quad{ }_{-}=\perp \\
& \llbracket \text { true } \rrbracket: \llbracket \text { Bool } \rrbracket \text { true true } \\
& \llbracket t r u e \rrbracket=t t \\
& \llbracket \text { false } \rrbracket: \llbracket \text { Bool } \rrbracket \text { false false } \\
& \llbracket \text { false } \rrbracket=t t
\end{aligned}
$$

(We use $T$ for nullary constructors as it is the identity of $\times$.)

parametricity and elimination Reynolds [1983] and Wadler [1989] assume that each type constant $K: \star$ is translated to the identity relation, as we have done for Bool above. This definition is certainly compatible with the condition required by Theorem 1 for such constants: $\llbracket K \rrbracket: \llbracket \star \rrbracket K K$, but so are many other relations. Are we missing some restriction for constants? This question might be answered by resorting to a translation to pure terms via Church encodings [Böhm and Berarducci 1985], as Wadler [2007] does. However, in the hope to shed a different light on the issue, we give another explanation, using our machinery.

Consider a base type, such as Bool : $\star$, equipped with constructors true : Bool and false : Bool. In order to derive parametricity theorems in a system containing such a constant Bool, we must define $\llbracket B o o l \rrbracket$, satisfying $\vdash \llbracket B o o l \rrbracket: \llbracket \star \rrbracket \overline{B o o l}$. What are the restrictions put on the term $\llbracket B o o l \rrbracket$ ? First, we must be able to define

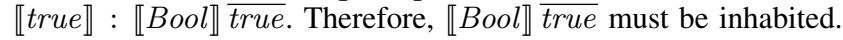
The same reasoning holds for the false case.

Second, to write any useful program using Booleans, a way to test their value is needed. This may be done by adding a constant if $:$ Bool $\rightarrow(A: \star) \rightarrow A \rightarrow A \rightarrow A$, such that if true $A x y \longrightarrow_{\beta} x$ and if false $A x y \longrightarrow_{\beta} y$. (This special case of Bool-elim is sufficient for the present example.)

Now, if a program uses if, we must also define $\llbracket$ if $\rrbracket$ of type $\llbracket B o o l \rightarrow(A: \star) \rightarrow A \rightarrow A \rightarrow A \rrbracket \overline{\text { if }}$ for parametricity to work. Let us expand the type of $\llbracket i f \rrbracket$ and attempt to give a definition case by case:

$$
\begin{aligned}
& \llbracket \text { if } \rrbracket:\left(b_{1} b_{2}: B o o l\right) \rightarrow\left(b_{R}: \llbracket B o o l \rrbracket b_{1} b_{2}\right) \rightarrow \\
& \left(A_{1} A_{2}: \star\right) \rightarrow\left(A_{R}: \llbracket \star \rrbracket A_{1} A_{2}\right) \rightarrow \\
& \left(x_{1}: A_{1}\right) \rightarrow\left(x_{2}: A_{2}\right) \rightarrow\left(x_{R}: A_{R} x_{1} x_{2}\right) \rightarrow \\
& \left(y_{1}: A_{1}\right) \rightarrow\left(y_{2}: A_{2}\right) \rightarrow\left(y_{R}: A_{R} y_{1} y_{2}\right) \rightarrow \\
& A_{R}\left(\text { if } b_{1} A_{1} x_{1} y_{1}\right)\left(\text { if } b_{2} A_{2} x_{2} y_{2}\right)
\end{aligned}
$$

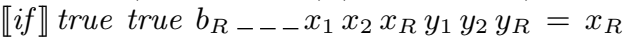

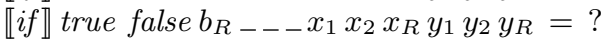

$$
\begin{aligned}
& \text { 【if false true } b_{R---} x_{1} x_{2} x_{R} y_{1} y_{2} y_{R}=\text { ? } \\
& \text { 【if』false false } b_{R---} x_{1} x_{2} x_{R} y_{1} y_{2} y_{R}=y_{R}
\end{aligned}
$$

(From this example onwards, we use a layout convention to ease the reading of translated types: each triple of arguments, corresponding to one argument in the original function, is written on its own line if space permits.)

In order to complete the above definition, we must provide a type-correct expression for each question mark. In the case of the second equation, this means that we must construct an expression of type $A_{R} x_{1} y_{2}$. Neither $x_{R}: A_{R} x_{1} x_{2}$ nor $y_{R}: A_{R} y_{1} y_{2}$ can help us here. The only liberty left is in $b_{R}: \llbracket B o o l \rrbracket$ true false. If we let $\llbracket B o o l \rrbracket$ true false be $\perp$, then this case can never be reached and we need not give an equation for it. This reasoning holds symmetrically for the third equation. Therefore, we have the restrictions:

$$
\begin{aligned}
& \llbracket B o o l \rrbracket x x=\text { some inhabited type } \\
& \llbracket B \text { ool } \rrbracket x y=\perp \quad \text { if } x \neq y
\end{aligned}
$$

We have some freedom regarding picking "some inhabited type", so we choose $\llbracket B o o l \rrbracket x x=\top$, yielding an encoding of the identity relation. In general, for any base type, the identity is the most permissive relation which allows for a definition of the translation of the eliminator.

An intuition behind parametricity is that, the more programs "know" about a type, the more restricted parametricity theorems are. Through the Bool example, we have seen how our framework captures this intuition, in a fine grained manner. We revisit this idea in Section 5.4.

lists and vectors From the definition of List in Figure 2, we have the constant List : $\star \rightarrow \star$, so List is an example of a type constructor, and thus $\llbracket L i s t \rrbracket$ is a relation transformer. The relation transformer we get by applying our scheme is exactly that given by Wadler [1989]: lists are related iff their lengths are equal and their elements are related point-wise.

$$
\begin{aligned}
& \llbracket \text { List } \rrbracket: \llbracket \star \rightarrow \star \rrbracket \text { List List } \\
& \llbracket \text { List } \rrbracket A_{1} A_{2} A_{R} \text { nil nil } \quad=\top \\
& \llbracket \text { List》 } A_{1} A_{2} A_{R}\left(\text { cons } x_{1} x s_{1}\right)\left(\text { cons } x_{2} x s_{2}\right)= \\
& A_{R} x_{1} x_{2} \times \llbracket L i s t \rrbracket A_{1} A_{2} A_{R} x s_{1} x s_{2} \\
& \llbracket \text { List } \rrbracket A_{1} A_{2} A_{R}-\quad \quad-\quad=\perp \\
& \llbracket n i l \rrbracket: \llbracket(A: \star) \rightarrow \text { List } A \rrbracket \text { nil nil } \\
& \llbracket n i l \rrbracket A_{1} A_{2} A_{R}=t t \\
& \llbracket \text { cons } \rrbracket: \llbracket(A: \star) \rightarrow A \rightarrow \text { List } A \rightarrow \text { List } A \rrbracket \text { cons cons } \\
& \llbracket \text { cons } \rrbracket A_{1} A_{2} A_{R} x_{1} x_{2} x_{R} x s_{1} x s_{2} x s_{R}=\left(x_{R}, x s_{R}\right)
\end{aligned}
$$

The translations of the constants of $V e c$ are given in Figure 3.

list rearrangements The first example of parametric type examined by Wadler [1989] is the type of list rearrangements: $R=(A$ : $\star) \rightarrow$ List $A \rightarrow$ List $A$. Intuitively, functions of type $R$ know nothing about the actual argument type $A$, and therefore they can only produce the output list by taking elements from the input list. In this section we recover that result as an instance of Theorem 1.

Applying the translation to $R$ yields:

$$
\begin{aligned}
& \llbracket R \rrbracket: R \rightarrow R \rightarrow \star \\
& \llbracket R \rrbracket r_{1} r_{2}=\left(A_{1} A_{2}: \star\right) \rightarrow\left(A_{R}: \llbracket \star \rrbracket A_{1} A_{2}\right) \rightarrow \\
& \left(l_{1}: \text { List } A_{1}\right) \rightarrow\left(l_{2}: \text { List } A_{2}\right) \rightarrow \\
& \left(l_{R}: \llbracket \text { List } A_{1} A_{2} A_{R} l_{1} l_{2}\right) \rightarrow \\
& \llbracket \text { List } A_{1} A_{2} A_{R}\left(r_{1} A_{1} l_{1}\right)\left(r_{2} A_{2} l_{2}\right)
\end{aligned}
$$

In words: two list rearrangements $r_{1}$ and $r_{2}$ are related iff for all types $A_{1}$ and $A_{2}$ with relation $A_{R}$, and for all lists $l_{1}$ and $l_{2}$ pointwise related by $A_{R}$, the resulting lists $r_{1} A_{1} l_{1}$ and $r_{2} A_{2} l_{2}$ are also point-wise related by $A_{R}$. By corollary 2 (parametricity), we have, for any $r$ :

$$
\vdash r: R \Longrightarrow \vdash \llbracket r \rrbracket: \llbracket R \rrbracket r r
$$




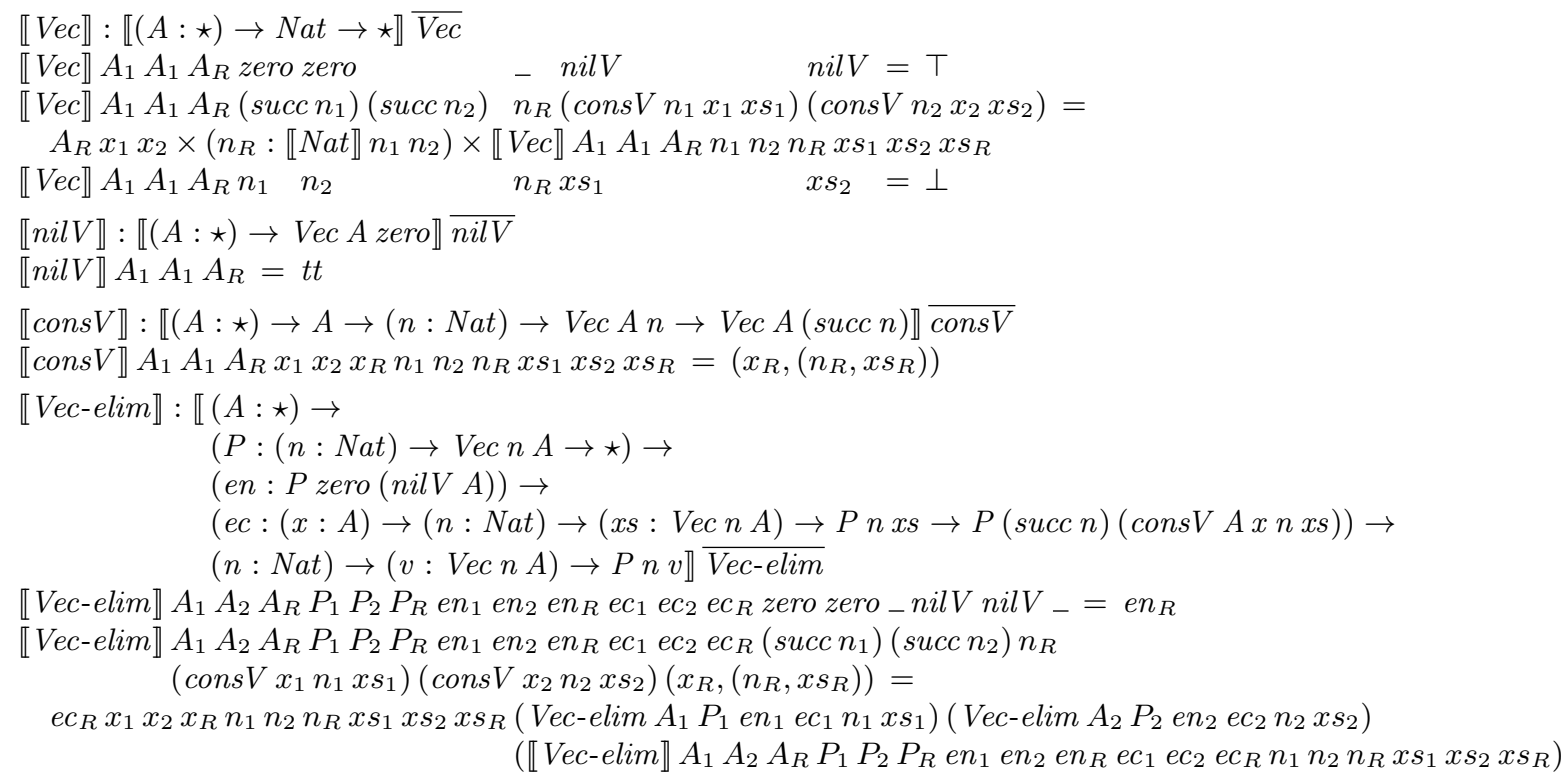

Figure 3. Deductive translation of $V e c$ constants. ( $\llbracket N a t \rrbracket$ is the identity relation.)

In words: applying $r$ preserves (point-wise) any relation existing between input lists. By specializing $A_{R}$ to a function $\left(A_{R} a_{1} a_{2}=\right.$ $f a_{1} \equiv a_{2}$ ) we obtain the well-known result:

$$
\begin{aligned}
& \vdash r: R \Longrightarrow \\
& \left(A_{1} A_{2}: \star\right) \rightarrow\left(f: A_{1} \rightarrow A_{2}\right) \rightarrow \\
& \left(l: \text { List } A_{1}\right) \rightarrow \\
& \quad \operatorname{map} f\left(r A_{1} l\right) \equiv r A_{2}(\operatorname{map} f l)
\end{aligned}
$$

(This form relies on the facts that $\llbracket$ List $\rrbracket$ preserves identities and composes with map.)

proof terms We have seen that applying II to a type yields a parametricity property for terms of that type. However, by Theorem 1 we can also apply $\llbracket-\rrbracket$ to a term of that type to obtain a proof of the property.

Consider a list rearrangement function odds that returns every second element from a list.

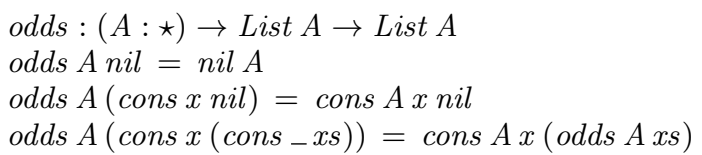

Any list rearrangement function must satisfy the parametricity condition seen above. We know by Theorem 1 that $\llbracket o d d s \rrbracket$ is a proof that $o d d s$ satisfies parametricity. Expanding it yields:

$$
\begin{aligned}
& \llbracket \text { odds } \rrbracket: \llbracket(A: \star) \rightarrow \text { List } A \rightarrow \text { List } A \rrbracket \text { odds odds } \\
& \llbracket \text { odds》 } A_{1} A_{2} A_{R} \text { nil nil }-=t t \\
& \llbracket \text { odds》 } A_{1} A_{2} A_{R}\left(\text { cons } x_{1} \text { nil }\right)\left(\operatorname{cons} x_{2} n i l\right)\left(x_{R},{ }_{-}\right)= \\
& \left(x_{R}, t t\right) \\
& \llbracket o d d s \rrbracket A_{1} A_{2} A_{R}\left(\text { cons } x_{1}\left(\text { cons } \_x s_{1}\right)\right) \\
& \left(\text { cons } x_{2}\left(\text { cons } \_x s_{2}\right)\right)\left(x_{R},\left(-, x s_{R}\right)\right)= \\
& \left(x_{R}, \llbracket o d d s \rrbracket A_{1} A_{2} A_{R} x s_{1} x s_{2} x s_{R}\right)
\end{aligned}
$$

We see that $\llbracket o d d s \rrbracket$ performs essentially the same computation as odds, on two lists in parallel. However, instead of building a new list, it keeps track of the relations (in the $R$-subscripted variables). This behaviour stems from the last two cases in the definition of $\llbracket o d d s \rrbracket$. Performing such a computation is enough to prove the parametricity condition.

\subsection{Inductive-style translation}

Inductive definitions offer another way of defining the translations $\llbracket c \rrbracket$ of the constants associated with a data type, an inductive definition in contrast to the deductive definitions of the previous section. Given an inductive family

$$
\begin{aligned}
& \operatorname{data} T(a: \mathrm{A}): \mathrm{K} \text { where } \\
& \quad c: \mathrm{C}
\end{aligned}
$$

by applying our translation to the components of the datadeclaration, we obtain an inductive family that defines the relational counterparts of the original type $T$ and its constructors $c$ at the same time:

$$
\begin{gathered}
\text { data } \llbracket T \rrbracket(\llbracket a: \mathrm{A} \rrbracket): \llbracket \mathrm{K} \rrbracket \overline{(T a)} \text { where } \\
c: \llbracket \mathrm{C} \rrbracket \overline{(c a)}
\end{gathered}
$$

It remains to supply a proof term for the parametricity of the elimination constant T-elim. If the inductive family has the form

$$
\begin{aligned}
& \text { data } T(a: \mathrm{A}): \forall n: \text { N. } s \text { where } \\
& c: \forall b: \mathrm{B} .(\forall x: \mathrm{X} . T a \mathrm{i}) \rightarrow T a \mathrm{v}
\end{aligned}
$$

then the proof $\llbracket T$-elim $\rrbracket$ can be defined using $\llbracket T \rrbracket$-elim and T-elim as follows:

$$
\begin{aligned}
& \llbracket T \text {-elim } \rrbracket: \llbracket \forall a: \text { A. } \forall P:(\forall n: \text { N. T a } n \rightarrow s) . \forall e: \text { Case }_{c} . \\
& \forall n: \text { N. } \forall t: T \text { a } n \text {. P } n t \rrbracket \overline{T-e l i m} \\
& \llbracket \text { T-elim a } P \text { e } \rrbracket=\llbracket T \rrbracket \text {-elim } \bar{a} a_{R} \\
& (\lambda \llbracket n: \mathrm{N}, t: T \text { a } n \rrbracket . \\
& \llbracket P n t \rrbracket \overline{(T \text {-elima Pent })}) \\
& (\lambda \llbracket b: \mathrm{B}, u:(\forall x: \text { X. Ta } \mathrm{i}) \rrbracket \text {. } \\
& \llbracket e b u \rrbracket \overline{(\lambda x: \mathrm{X} . T \text {-elima Pei }(u x))}
\end{aligned}
$$

Deductive and inductive-style translations define the same relation, but the objects witnessing the instances of the inductively definedrelation record additional information, namely which rules are used to prove membership of the relation. However, since the same constructor never appears in more than one case of the inductive definition, that additional content can be recovered from a witness of the deductive-style; therefore the two styles are truly isomorphic. 
Booleans Applying the above scheme to the data-declaration of Bool (from Figure 2), we obtain:

$$
\begin{gathered}
\text { data } \llbracket \text { Bool } \rrbracket: \llbracket \star \rrbracket \overline{\text { Bool }} \text { where } \\
\text { 【rue } \rrbracket: \llbracket \text { Bool } \rrbracket \text { true } \\
\llbracket \text { false } \rrbracket: \llbracket \text { Bool } \rrbracket \text { false }
\end{gathered}
$$

The main difference from the deductive-style definition is that it is possible, by analysis of a value of type $\llbracket B o o l \rrbracket$, to recover the arguments of the relation (either all true, or all false).

The elimination constant for $\mathrm{Bool}$ is

$$
\begin{aligned}
\text { Bool-elim }: & (P: \text { Bool } \rightarrow \star) \rightarrow P \text { true } \rightarrow P \text { false } \rightarrow \\
& (b: \text { Bool }) \rightarrow P b
\end{aligned}
$$

Similarly, our new type $\llbracket B o o l \rrbracket$ (with $n=2$ ) has an elimination constant with the following type:

$$
\begin{aligned}
& \llbracket \text { Bool } \rrbracket \text {-elim }: \\
& \quad\left(C:\left(a_{1} a_{2}: \text { Bool }\right) \rightarrow \llbracket \text { Bool } \rrbracket a_{1} a_{2} \rightarrow \star\right) \rightarrow \\
& \quad C \text { true true } \llbracket \text { true } \rrbracket \rightarrow C \text { false false } \llbracket \text { false } \rrbracket \rightarrow \\
& \quad\left(b_{1} b_{2}: \text { Bool }\right) \rightarrow\left(b_{R}: \llbracket \text { Bool } \rrbracket b_{1} b_{2}\right) \rightarrow C b_{1} b_{2} b_{R}
\end{aligned}
$$

As an instance of the above scheme, we can define $\llbracket$ Bool-elim $\rrbracket$ using the elimination constants $\llbracket B o o l \rrbracket$ and $\llbracket B o o l \rrbracket$-elim as follows (where $t=$ true and $f=$ false):

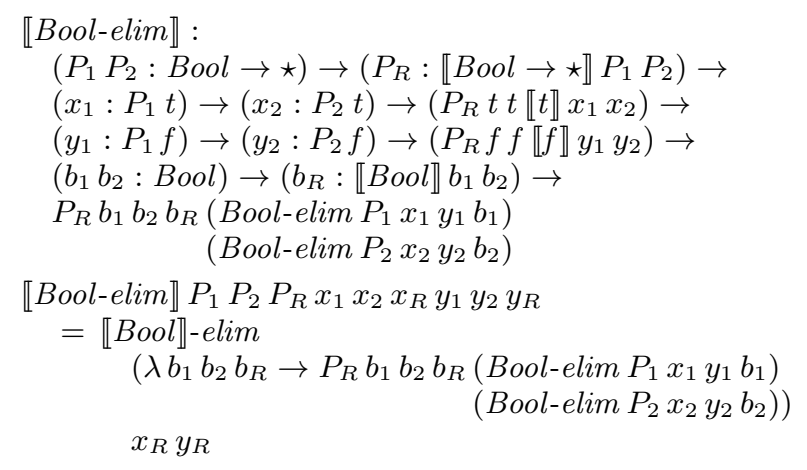

lists For List, as introduced in Figure 2, we have the following translation:

$$
\begin{gathered}
\text { data } \llbracket \text { List } \rrbracket(\llbracket A: \star \rrbracket): \llbracket \star \rrbracket \overline{(\text { List } A)} \text { where } \\
\llbracket n i l \rrbracket: \llbracket \text { List } A \rrbracket(\text { nil } A) \\
\llbracket \text { cons } \rrbracket: \llbracket A \rightarrow \text { List } A \rightarrow \text { List } A \rrbracket \overline{(\operatorname{cons} A)}
\end{gathered}
$$

or after expansion (for $n=2$ ):

$$
\begin{gathered}
\text { data } \llbracket \text { List }\left(A_{1} A_{2}: \star\right)\left(A_{R}: \llbracket \star \rrbracket A_{1} A_{2}\right): \\
\text { List } A_{1} \rightarrow \text { List } A_{2} \rightarrow \star \text { where } \\
\llbracket n i l \rrbracket: \llbracket \text { List } \rrbracket A_{1} A_{2} A_{R}\left(\text { nil } A_{1}\right)\left(\text { nil } A_{2}\right) \\
\llbracket \text { cons } \rrbracket:\left(x_{1}: A_{1}\right) \rightarrow\left(x_{2}: A_{2}\right) \rightarrow\left(x_{R}: A_{R} x_{1} x_{2}\right) \rightarrow \\
\left(x s_{1}: \text { List } A_{1}\right) \rightarrow\left(x s_{2}: \text { List } A_{2}\right) \rightarrow \\
\left(x s_{R}: \llbracket \text { List } \rrbracket A_{1} A_{2} A_{R} x s_{1} x s_{2}\right) \rightarrow \\
\llbracket \text { List } \rrbracket A_{1} A_{2} A_{R}\left(\text { cons } A_{1} x_{1} x s_{1}\right) \\
\left(\text { cons } A_{2} x_{2} x s_{2}\right)
\end{gathered}
$$

The above definition encodes the same relational action as that given in Section 4.2. Again, the difference is that the derivation of a relation between lists $l_{1}$ and $l_{2}$ is available as an object of type $\llbracket L i s t \rrbracket A_{1} A_{2} A_{R} l_{1} l_{2}$.

proof terms The proof term for the list-rearrangement example can be constructed in a similar way to the inductive one. The main difference is that the target lists are also built and recorded in the $\llbracket L i s t \rrbracket$ structure. In short, this version has more of a computational flavour than the inductive version.

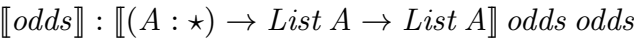

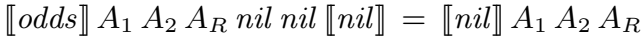

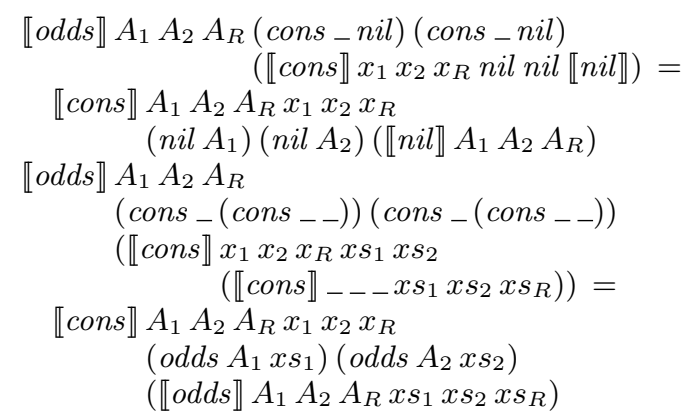

vectors We can apply the same translation method to inductive families. For example, Figures 4 and 5 give the translation of the family $V e c$, corresponding to lists indexed by their length. The relation obtained by applying «-』 encodes that vectors are related if their lengths are the same and if their elements are related pointwise. The difference with the List version is that the equality of lengths is encoded in $\llbracket c o n s V \rrbracket$ as a Nat (identity) relation.

\section{Applications}

In this section we shall see how examples going beyond Wadler [1989] can be expressed in our setting. All examples fit within the system $I_{\omega}$ augmented with inductive definitions.

\subsection{Type classes}

What if a function is not parametrized over all types, but only types equipped with decidable equality? One way to model this difference in a pure type system is to add an extra parameter to capture the extra constraint. For example, a function $n u b: N u b$ removing duplicates from a list may be given the following type:

$$
N u b=(A: \star) \rightarrow E q A \rightarrow \text { List } A \rightarrow \text { List } A
$$

The equality requirement itself may be modelled as a mere comparison function: $E q A=A \rightarrow A \rightarrow B o o l$. In that case, the parametricity statement is amended with an extra requirement on the relation between types, which expresses that $e q_{1}$ and $e q_{2}$ must respect the $A_{R}$ relation. Formally:

$$
\begin{aligned}
& \llbracket E q A \rrbracket e q_{1} e q_{2}=\left(a_{1}: A_{1}\right) \rightarrow\left(a_{2}: A_{2}\right) \rightarrow A_{R} a_{1} a_{2} \rightarrow \\
& \left(b_{1}: A_{1}\right) \rightarrow\left(b_{2}: A_{2}\right) \rightarrow A_{R} b_{1} b_{2} \rightarrow \\
& \llbracket B o o l \rrbracket\left(e q_{1} a_{1} b_{1}\right)\left(e q_{2} a_{2} b_{2}\right) \\
& \llbracket N u b \rrbracket n_{1} n_{2}= \\
& \left(A_{1} A_{2}: \star\right) \rightarrow\left(A_{R}: \llbracket \star \rrbracket A_{1} A_{2}\right) \rightarrow \\
& \left(e q_{1}: E q A_{1}\right) \rightarrow\left(e q_{2}: E q A_{2}\right) \rightarrow \llbracket E q A \rrbracket e q_{1} e q_{2} \rightarrow \\
& \left(l_{1}: \text { List } A_{1}\right) \rightarrow\left(l_{2}: \text { List } A_{2}\right) \rightarrow \llbracket \text { List } A \rrbracket l_{1} l_{2} \rightarrow \\
& \llbracket \text { List } A \rrbracket\left(n_{1} A_{1} e q_{1} l_{1}\right)\left(n_{2} A_{2} e q_{2} l_{2}\right)
\end{aligned}
$$

So far, this is just confirming the informal description in Wadler [1989]. But with access to full dependent types, one might wonder: what if we model equality more precisely, for example by requiring $e q$ to be reflexive?

$$
\begin{aligned}
& E q^{\prime} A=(e q: A \rightarrow A \rightarrow B o o l) \times \text { Refl eq } \\
& \text { Refl eq }=(x: A) \rightarrow \text { eq } x x \equiv \text { true }
\end{aligned}
$$

In the case of $E q^{\prime}$, the parametricity condition does not become more exciting. It merely requires the proofs of reflexivity at $A_{1}, A_{2}$ to be related. This extra condition adds nothing new: since there is at most one element in (and thus proof of) $x \equiv y$, one already expects proofs to be related.

The observations drawn from this simple example can be generalized in two ways. First, proof arguments do not strengthen parametricity conditions in useful ways. One often does not care about the actual proof of a proposition, but merely that it exists, so knowing that two proofs are related adds nothing. Secondly, type-classes 


$$
\begin{aligned}
& \text { data } \llbracket \operatorname{Vec} \rrbracket(\llbracket A: \star \rrbracket): \llbracket N a t \rightarrow \star \rrbracket \overline{(\operatorname{Vec} A)} \text { where } \\
& \llbracket n i l V \rrbracket: \llbracket \text { Vec A zero } \rrbracket \overline{(n i l V A)} \\
& \llbracket c o n s V \rrbracket: \llbracket(x: A) \rightarrow(n: N a t) \rightarrow \operatorname{Vec} A n \rightarrow \operatorname{Vec} A(\text { succ } n) \rrbracket \overline{(\text { cons } V A)} \\
& \text { data } \llbracket V e c \rrbracket\left(A_{1} A_{2}: \star\right)\left(A_{R}: A_{1} \rightarrow A_{2} \rightarrow \star\right):\left(n_{1} n_{2}: N a t\right) \rightarrow\left(n_{R}: \llbracket N a t \rrbracket n_{1} n_{2}\right) \rightarrow \\
& \operatorname{Vec} A_{1} n_{1} \rightarrow \operatorname{Vec} A_{2} n_{2} \rightarrow \star \text { where } \\
& \llbracket n i l V \rrbracket: \llbracket V e c \rrbracket A_{1} A_{2} A_{R} \text { zero zero } \llbracket z e r o \rrbracket\left(\text { nilV } A_{1}\right)\left(\text { nilV } A_{2}\right) \\
& \llbracket \text { cons } V \rrbracket:\left(x_{1}: A_{1}\right) \rightarrow\left(x_{2}: A_{2}\right) \rightarrow\left(x_{R}: A_{R} x_{1} x_{2}\right) \rightarrow \\
& \left(n_{1}: N a t\right) \rightarrow\left(n_{2}: N a t\right) \rightarrow\left(n_{R}: \llbracket N a t \rrbracket n_{1} n_{2}\right) \rightarrow \\
& \left(x s_{1}: \operatorname{Vec} A_{1} n_{1}\right) \rightarrow\left(x s_{2}: \operatorname{Vec} A_{2} n_{2}\right) \rightarrow\left(x s_{R}: \llbracket V e c \rrbracket A_{1} A_{2} A_{R} n_{1} n_{2} n_{R} x s_{1} x s_{2}\right) \rightarrow \\
& \llbracket V e c \rrbracket A_{1} A_{2} A_{R}\left(\text { succ }_{1}\right)\left(\text { succ }_{2}\right)\left(\llbracket \text { succ } \rrbracket n_{1} n_{2} n_{R}\right)\left(\text { consV } A_{1} x_{1} n_{1} x s_{1}\right)\left(\operatorname{consV} A_{2} x_{2} n_{2} x s_{2}\right)
\end{aligned}
$$

Figure 4. Inductive translation of $V e c$, both before and after expansion.

$$
\begin{aligned}
& \llbracket \text { Vec-elim } \rrbracket: \llbracket(A: \star) \rightarrow \\
& (P:(n: N a t) \rightarrow \operatorname{Vec} n A \rightarrow \star) \rightarrow \\
& (\text { en }: P \text { zero }(\text { nilV } A)) \rightarrow \\
& (\text { ec }:(x: A) \rightarrow(n: N a t) \rightarrow(x s: \operatorname{Vec} n A) \rightarrow P n x s \rightarrow P(\text { succ } n)(\text { consV A } x n x s)) \rightarrow \\
& (n: N a t) \rightarrow(v: \operatorname{Vec} n A) \rightarrow P n v \rrbracket \overline{\text { Vec-elim }} \\
& (\lambda \llbracket n: N a t, v: \operatorname{Vec} n A \rrbracket \rightarrow \llbracket P n v \rrbracket \overline{(\text { Vec-elim A P en ec } v)}) \\
& e n_{R} \\
& (\lambda \llbracket x: A, n: N a t, x s: \operatorname{Vec} n A \rrbracket \rightarrow \llbracket e c x n x s \rrbracket \overline{(\text { Vec-elim A P en ec xs })})
\end{aligned}
$$

Figure 5. Proof term for Vec-elim using the inductive-style definitions.

may be encoded as their dictionary of methods [Wadler and Blott 1989]. Indeed, even if a type class has associated laws, they have little impact on the parametricity results.

\subsection{Constructor classes}

Having seen how to apply our framework both to type constructors and type classes, we now apply it to types quantified over a type constructor, with constraints.

Voigtländer [2009b] provides many such examples, using the Monad constructor class. They fit well in our framework. For the sake of brevity, we do not detail them more here. We can however detail the definition of the simpler Functor class, which can be modelled as follows:

$$
\begin{aligned}
\text { Functor }= & (F: \star \rightarrow \star) \times \\
& ((X Y: \star) \rightarrow(X \rightarrow Y) \rightarrow F X \rightarrow F Y)
\end{aligned}
$$

Our translation readily applies to the above definition, and yields the following relation between functors:

$$
\begin{aligned}
& \llbracket \text { Functor } \rrbracket\left(F_{1}, \text { map }_{1}\right)\left(F_{2}, \text { map }_{2}\right) \\
& =\left(F_{R}:\left(A_{1} A_{2}: \star\right) \rightarrow\left(A_{R}: A_{1} \rightarrow A_{2} \rightarrow \star\right) \rightarrow\right. \\
& \left.\left(F_{1} A_{1} \rightarrow F_{2} A_{2} \rightarrow \star\right)\right) \times \\
& \left(\left(X_{1} X_{2}: \star\right) \rightarrow\left(X_{R}: X_{1} \rightarrow X_{2} \rightarrow \star\right) \rightarrow\right. \\
& \left(Y_{1} Y_{2}: \star\right) \rightarrow\left(Y_{R}: Y_{1} \rightarrow Y_{2} \rightarrow \star\right) \rightarrow \\
& \left(f_{1}: X_{1} \rightarrow Y_{1}\right) \rightarrow\left(f_{2}: X_{2} \rightarrow Y_{2}\right) \rightarrow \\
& \left(\left(x_{1}: X_{1}\right) \rightarrow\left(x_{2}: X_{2}\right) \rightarrow\left(x_{R}: X_{R} x_{1} x_{2}\right) \rightarrow\right. \\
& \left.Y_{R}\left(f_{1} x_{1}\right)\left(f_{2} x_{2}\right)\right) \rightarrow \\
& \left(y_{1}: F_{1} X_{1}\right) \rightarrow\left(y_{2}: F_{2} X_{2}\right) \rightarrow \\
& \left(y_{R}: F_{R} X_{R} y_{1} y_{2}\right) \rightarrow \\
& \left.F_{R} Y_{R}\left(\operatorname{map}_{1} f_{1} y_{1}\right)\left(\operatorname{map}_{2} f_{2} y_{2}\right)\right)
\end{aligned}
$$

In words, the translation of a functor is the product of a relation transformer $\left(F_{R}\right)$ between functors $F_{1}$ and $F_{2}$, and a witness $\left(\operatorname{map}_{R}\right)$ that $\mathrm{map}_{1}$ and $\mathrm{map}_{2}$ preserve relations.
Such Functors can be used to define a generic fold operation, which typically takes the following form:

$$
\begin{aligned}
& \text { data } \mu((F, \text { map }): \text { Functor }): \star \text { where } \\
& \quad \text { In }: F(\mu(F, \text { map })) \rightarrow \mu(F, \text { map }) \\
& \text { fold }:((F, \text { map }): \text { Functor }) \rightarrow(A: \star) \rightarrow \\
& \quad(F A \rightarrow A) \rightarrow \mu(F, \text { map }) \rightarrow A \\
& \text { fold }(F, \text { map }) A \phi(\text { In } d)= \\
& \phi(\text { map }(\mu(F, \text { map })) A(\text { fold }(F, \text { map }) A \phi) d)
\end{aligned}
$$

Note that the $\mu$ datatype is not strictly positive, so its use would be prohibited in many dependently-typed languages to avoid inconsistency. However, if one restricts oneself to well-behaved functors (yielding strictly positive types), then consistency is restored both in the source and target systems, and the parametricity condition derived for fold is valid.

One can see from the type of fold that it behaves uniformly over $(F$, map $)$ as well as $A$. By applying 【-』 to fold and its type, this observation can be expressed (and justified) formally and used to reason about fold. Further, every function defined using fold, and in general any function parametrized over any functor enjoys the same kind of property.

Gibbons and Paterson [2009] previously made a similar observation in a categorical setting, showing that fold is a natural transformation between higher-order functors. Their argument heavily relies on categorical semantics and the universal property of fold, while our type-theoretical argument uses the type of fold as a starting point and directly obtains a parametricity property. However some additional work is required to obtain the equivalent property using natural transformations and horizontal compositions from the parametricity property.

\subsection{Generic cast}

Continuing to apply our framework to terms of increasingly rich types, the next candidate is dependently typed. 
An important application of dependent types is that of generic programming with universes, as in the work of Altenkirch and McBride [2003]; Benke et al. [2003]. The basic idea is to represent the "universe" of types as data, and provide an interpretation function from values of this data type to types (in $\star$ ). Generic functions can then be written by pattern matching on the type representation. While universes usually capture large classes of types, we use as an example a very simple universe of types for Booleans and natural numbers, as follows. ${ }^{4}$

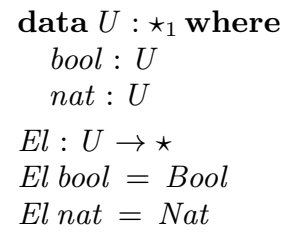

An example of a dependently-typed, generic function is gcast, which for any type context $F$ and any two (codes for) types $u$ and $t$, returns a casting function between $F(E l u)$ and $F(E l t)$, if $u$ and $t$ are the same (and nothing otherwise).

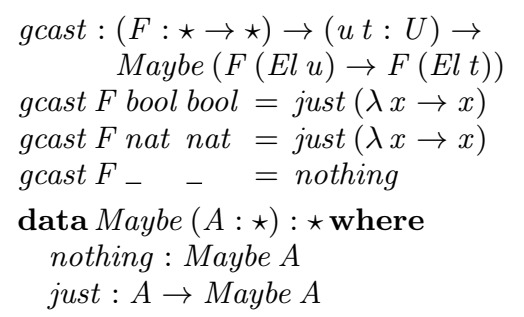

The function gcast is deemed safe if it returns the identity function whenever it returns something. Vytiniotis and Weirich [2009] show that this theorem can be deduced from the type of gcast alone, by parametricity. While the result can be re-derived in a simple way by reasoning directly on the definition of gcast, there is a good reason for using parametricity: as the universe is extended to a realistic definition, the definition of gcast gets more complex, but its type remains the same, and therefore the argument relying on parametricity is unchanged.

The rest of this section is devoted to rederiving the result using our framework. The first step is to encode the theorem. We can encode that an arbitrary function $f: A \rightarrow B$ is the identity as the formula $(x: A) \rightarrow f x \cong x$. Note that because the input and output types of the cast are not definitionally equal, we must use a heterogeneous equality $(\cong)$, defined as follows:

$$
\begin{aligned}
& \text { data } \cong(A: \star)(a: A):(B: \star) \rightarrow B \rightarrow \star \text { where } \\
& \quad \text { refl } f^{\prime}: \cong A \text { a } A a
\end{aligned}
$$

Now, gcast is not a direct conversion function: sometimes it returns no result; its result is wrapped in Maybe. Hence we use a helper function to lift the identity predicate to a Maybe type:

$$
\begin{aligned}
& \text { onMaybe }:(A: \star) \rightarrow(A \rightarrow \star) \rightarrow \text { Maybe } A \rightarrow \star \\
& \text { onMaybe } A \text { P nothing }=\top \\
& \text { onMaybe } A P(\text { just } a)=P a
\end{aligned}
$$

The theorem can then be expressed as follows:

Theorem 3 (gcast is safe).

$$
\begin{gathered}
(F: \star \rightarrow \star) \\
\rightarrow(u t: U) \rightarrow(x: F(\text { El } u)) \rightarrow \\
\text { onMaybe }(F(\text { El } u) \rightarrow F(E l t)) \\
(\lambda \text { cast } \rightarrow \text { cast } x \cong x) \\
(\text { gcast } F u t)
\end{gathered}
$$

\footnotetext{
${ }^{4}$ For the present section, $U: \star$ would be sufficient, but we define $U: \star 1$ to permit a different definition of $\llbracket U \rrbracket$ in the next section.
}

We remark that onMaybe is in fact the deductive version of

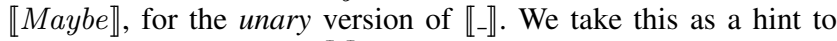
use the unary version of $\llbracket-\rrbracket$, and derive relations of the following types:

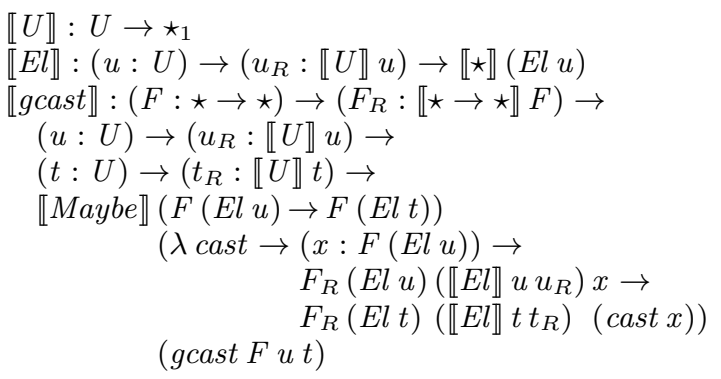

Additionally, we can define $\operatorname{param}_{U}$ :

$$
\begin{aligned}
& \operatorname{param}_{U}:(u: U) \rightarrow \llbracket U \rrbracket u \\
& \operatorname{param}_{U} \text { bool }=\llbracket \text { bool } \rrbracket \\
& \operatorname{param}_{U} \text { nat }=\llbracket n a t \rrbracket
\end{aligned}
$$

We can then use $\llbracket$ gcast $\rrbracket$ to prove the theorem. The idea is to specialize it to the types and relations of interest:

$$
\begin{aligned}
& \text { lemma1 }:(F: \star \rightarrow \star) \rightarrow(u t: U) \rightarrow(x: F(E l u)) \rightarrow \\
& \llbracket \text { Maybe } \rrbracket(F(E l u) \rightarrow F(E l t)) \\
&\left(\lambda \text { cast } \rightarrow\left(x^{\prime}: F(E l u)\right) \rightarrow x^{\prime} \cong x \rightarrow\right.\text { cast } \left.x^{\prime} \cong x\right) \\
&(\text { gcast } F u t) \\
& \text { lemma1 } F u t x=\llbracket \text { gcast } \rrbracket F\left(\lambda t_{R} y \rightarrow y \cong x\right) \\
& u\left(\text { param }_{U} u\right) \\
& t\left(\text { param }_{U} t\right)
\end{aligned}
$$

By fixing $x^{\prime}$ to $x$ in the argument to $\llbracket$ Maybe $\rrbracket$, the condition $x^{\prime} \cong x$ is fulfilled, and the proof is complete.

The remarkable feature of this proof is that it is essentially independent of the definitions of $U$ and $E l$ : only their types matter. Adding constructors in $U$ would not change anything in the proof: $\llbracket$ gcast $\rrbracket$ isolates Theorem 3 from the actual definitions of $U, E l$ and gcast; it can be generated automatically from gcast. steps:

In summary, we have proved the correctness of gcast in three

1. Model representation types within our dependently-typed language;

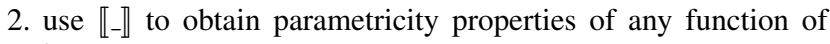
interest;

3. prove correctness by using the properties.

We think that the above process is an economical way to work with parametricity for extended type systems. Indeed, step one of the above process is becoming an increasingly popular way to develop languages with exotic type systems as an embedding in a dependently-typed language [Oury and Swierstra 2008]. By providing (an automatic) step two, we hope to spare language designers the effort to adapt Reynolds' abstraction theorem for new type systems in an ad-hoc way.

\subsection{A partially constrained universe}

So far we have only seen universes which are either completely unconstrained (like $\star$ ) and translate to arbitrary relations, or universes which are completely constrained (like Bool or $U$ in the previous section) and translate to the identity relation. In this section we show that a middle ground is also possible.

Suppose that we want the same universe as in the above section, but with only limited capabilities to dispatch on the type. That is, we allow users to define functions that have special behaviour for 
Booleans, but are otherwise oblivious to the actual type at which they are used. This particular functionality may be encoded by only providing an eliminator for $U$ with restricted capabilities:

$$
\begin{aligned}
\text { type Test }:(u: U) \rightarrow(F: \star \rightarrow \star) \rightarrow \\
F \text { Bool } \rightarrow((A: \star) \rightarrow F A) \rightarrow F(E l u) \\
\text { typeTest bool } F A_{B} A_{G e n}=A_{B} \\
\text { typeTest } t \quad F A_{B} A_{G e n}=A_{G e n}(E l t)
\end{aligned}
$$

This restriction of elimination allows us to "relax" the definitions of $\llbracket U \rrbracket$ and $\llbracket E l \rrbracket$, by translating the cases that do not involve bool to an arbitrary relation (for $n=2$ ):

$$
\begin{aligned}
& \llbracket U \rrbracket: U \rightarrow U \rightarrow \star_{1} \\
& \llbracket U \rrbracket \text { bool bool }=\top \\
& \llbracket U \rrbracket \text { bool }_{-}=\perp \\
& \llbracket U \rrbracket \_ \text {bool }=\perp \\
& \llbracket U \rrbracket u_{1} \quad u_{2}=\llbracket \star \rrbracket\left(E l u_{1}\right)\left(E l u_{2}\right) \\
& \llbracket E l \rrbracket:\left(u_{1} u_{2}: U\right) \rightarrow\left(u_{R}: \llbracket U \rrbracket u_{1} u_{2}\right) \rightarrow \\
& \llbracket \star \rrbracket\left(E l u_{1}\right)\left(E l u_{2}\right) \\
& \llbracket E l \rrbracket \text { bool bool } r=\llbracket B o o l \rrbracket \\
& \llbracket E l \rrbracket u_{1} \quad u_{2} \quad r=r
\end{aligned}
$$

Given the above definitions, free theorems involving $U$ reduce to the constrained case if presented with Booleans, and to the unconstrained case otherwise.

While the above is a toy example, it points the way towards more sophisticated representations of universes. An example would be an encoding of fresh abstract type variables, as in Neis et al. [2009].

\section{Discussion \\ 6.1 Proof}

A detailed sketch of the proof of Theorem 1 is available online [Bernardy et al. 2010b]. Beyond the pen-and-paper version, we also have a machine-checked proof, for the unary case, as an Agda program [Bernardy 2010]. A few improvements are necessary before it can be considered a fully-machine-checked proof:

- some substitution lemmas need to be proved;

- the top-level structure needs some superficial restructuring to pass the termination-check of the Agda system;

- proofs of some lemmas given by Barendregt [1992] should be formalized.

\subsection{Different source and target sorts}

Even though the sort-mapping function _ used in all our examples has been the identity, there are other possible choices. For example, $I_{\omega}$ is reflective with $\widetilde{\star}_{i}=\star_{i+k}$, for any natural $k$. Other examples can be constructed by mapping $\_$to "fresh" sorts. The following system $\left(I_{\omega}{ }^{+}\right)$is reflective with $\widetilde{\star_{i}}=\triangle_{i}$ and $\widetilde{\triangle_{i}}=\triangle_{i}$.

Definition $7\left(I_{\omega}^{+}\right) . I_{\omega}^{+}$is a PTS with this specification:

$$
\begin{aligned}
\text { - } \mathcal{S}= & \left\{\star_{i} \mid i \in \mathbb{N}\right\} \cup\left\{\triangle_{i} \mid i \in \mathbb{N}\right\} \\
\text { - } \mathcal{A}= & \left\{\triangle_{i}: \triangle_{i+1} \mid i \in \mathbb{N}\right\} \cup\left\{\star_{i}: \star_{i+1} \mid i \in \mathbb{N}\right\} \\
\text { - } \mathcal{R}= & \left\{\left(\star_{i}, \star_{j}, \star_{\max (i, j)}\right) \mid i, j \in \mathbb{N}\right\} \\
& \left\{\left(\triangle_{i}, \triangle_{j}, \triangle_{\max (i, j)}\right) \mid i, j \in \mathbb{N}\right\} \\
& \left\{\star_{i} \leadsto \triangle_{j} \mid i \leqslant j \in \mathbb{N}\right\}
\end{aligned}
$$

\subsection{Different source and target systems}

For simplicity, we have chosen to use the same source and target PTS in Theorem 1. However, the theorem may be generalized to the case where source and target are different. One way to relax the hypothesis is to allow any source PTS which is a subsystem of the target one, keeping the same conditions for the target PTS.
For example, using this generalization, we see that all the parametricity statements about terms in the $\lambda$-cube are expressible and provable in the generalized calculus of constructions $\left(\mathrm{CC}_{\omega}\right)$. Indeed, we observe that

- $\mathrm{CC}_{\omega}$ is reflective with $\widetilde{s}=s$ and,

- All eight systems of the $\lambda$-cube are embedded in $\mathrm{CC}_{\omega}$

While extending our abstraction to subsystems is useful, further generalization is possible. For example, parametricity theorems (and proofs) generated from terms in the $\lambda$-cube will never use the higher sorts of $\mathrm{CC}_{\omega}$. Specifying necessary and sufficient conditions for the two-system case is left as future work.

\subsection{Internalizing the meta-theorem}

Theorem 1 and Corollary $2(\vdash A: B \Longrightarrow \vdash \llbracket A \rrbracket: \llbracket B \rrbracket \bar{A})$ are meta-theorems. One can instantiate the corollary by choosing specific terms $A$ and $B$; then $\llbracket A \rrbracket$ is a proof of $\llbracket B \rrbracket \bar{A}$ in the system, derived from the structure of $\vdash A$ : $B$. Our examples consist of many such instantiations.

However, one would like to go further and make a general statement about about all values of type $B$ within the system. That is, for a type $\mathrm{B}$, to define $\operatorname{param}_{\mathrm{B}}:(\forall x: \mathrm{B}$. $\llbracket \mathrm{B} \rrbracket x \ldots x)$, as we did with param $_{U}$ in Section 5.3, essentially making the semantics of the type available for reasoning within the system. In particular, for any constant $k: \mathrm{B}$, we could define $\llbracket k \rrbracket=\operatorname{param}_{\mathrm{B}} k$.

One way to proceed is to assert parametricity at all types, with a constant param $_{\mathrm{B}}$ for each B. This approach was applied to CC by Takeuti [2004], extending similar axiom schemes for System $\mathrm{F}$ by Plotkin and Abadi [1993]. For each $\alpha: \square$ and $\mathrm{P}: \alpha$, Takeuti defined a relational interpretation $\langle\mathrm{P}\rangle$ and a kind $(\mathrm{P}: \alpha)$ such that $\langle\mathrm{P}\rangle:(\mathrm{P}: \alpha)$. Then for each type $\mathrm{T}: \star$, he postulated an axiom $\operatorname{param}_{\mathrm{T}}:(\forall x: \mathrm{T} .\langle\mathrm{T}\rangle x x)$, conjecturing that such axioms did not make the system inconsistent. For closed terms $P$, Takeuti's translations $\langle\mathrm{P}\rangle$ and $(\mathrm{P}: \alpha)$ resemble our $\llbracket \mathrm{P} \rrbracket$ and $\llbracket \alpha \rrbracket \overrightarrow{\mathrm{P}}$ respectively (with $n=2$ ), but the pattern is obscured by an error in the translation rule for the product $\square \sim \star$, and the omission of a witness $x_{R}$ for the relationship between values $x_{1}$ and $x_{2}$ in the rules corresponding to the product $\star \sim \square$.

Another approach would be to provide access to the terms via some form of reflection.

\subsection{Related work}

Some of the many studies of parametricity have already been mentioned and analysed in the rest of the paper. In this section we compare our work to only a couple of the most relevant pieces of work.

One direction of research is concerned with parametricity in extensions of System F. Our work is directly inspired by Vytiniotis and Weirich [2010], which extend parametricity to (an extension of) $\mathrm{F} \omega$ : indeed, $\mathrm{F} \omega$ can be seen as a PTS with one more product rule than System F.

Besides supporting more sorts and function spaces, an orthogonal extension of parametricity theory is to support impure features in the system. For example, [Johann and Voigtländer 2005] studied how explicit strictness modifies parametricity results. It is not obvious how to support such extensions in our framework.

Another direction of research is concerned with better understanding of parametricity. Here we shall mention only [Wadler 2007], which gives a particularly lucid presentation of the abstraction theorem, as the inverse of Girard's Representation theorem [Girard 1972]. Our version of the abstraction theorem differs in the following aspects compared to that of Wadler (and to our knowledge all others):

1. Instead of targeting a logic, we target its propositions-as-types interpretation, expressed in a PTS. 
2. We abstract from the details of the systems, generalizing to a class of PTS's.

3. We add that the translation function used to interpret types as relations can also be used to interpret terms as witnesses of those relations. In short, the $\llbracket A \rrbracket$ part of $\Gamma \vdash A: B \Longrightarrow$ $\llbracket \Gamma \rrbracket \vdash \llbracket A \rrbracket: \llbracket B \rrbracket \bar{A}$ is new. This additional insight depends heavily on using the propositions-as-types interpretation.

It also appears that the function $\llbracket-\rrbracket$ (for the unary case) has been discovered independently by Monnier and Haguenauer [2010], for

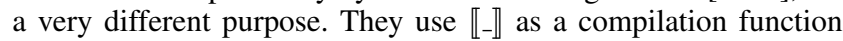
from $\mathrm{CC}$ to a language with singleton types only, in order to enforce phase-distinction. Type preservation of the translation scheme is the main formal property presented by Monnier and Haguenauer. We remark that this property corresponds to the abstraction theorem for CC.

\subsection{Future work}

Our explanation of parametricity for dependent types has opened a whole range of interesting topics for future work.

We should investigate whether our framework can be applied (and extended if need be) to more exotic systems, for example those incorporating strictness annotations $(s e q)$ or non-termination.

We should extend our translation to support non-informative function spaces, as found for example in Coq. In Coq, the sort $\star$ of $\mathrm{CC}$ is split into two separate sorts, one for types $(\mathrm{Set})$ and one for propositions (Prop). Inhabitants of Set can depend on inhabitants of Prop: for example, a program may depend on a certain property to terminate. However, computational content can never "leak" from Prop to Set: programs may only depend on the existence of a proof; it is forbidden to inspect their structure. In such a situation, our translation scheme appears to generate parametricity results that are too weak, as we have briefly alluded to in Section 5.1. The reason is that we always assume that computational content may be transferred from the argument of a function to its result. We could modify the translation to omit the superfluous relation parameter in such cases.

Reynolds' abstraction theorem can be understood as an embedding of polymorphic lambda calculus into second order propositional logic. Wadler [2007] showed that Girard's representation theorem [Girard 1972] can be understood as the corresponding projection. In this work we have shown that the embedding can be generalized for more complex type systems. The question of how the projection generalizes naturally arises, and should also be addressed.

It is straightforward to derive translated types using our schema,

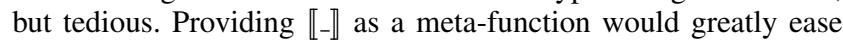
experimentation with our technique. Another direction worth exploring is to provide the parametricity axiom (param_) as a metafunction in a logical framework.

We presented only simple examples. Applying the results to more substantial applications should be done as well.

\section{Conclusion}

We have shown that it is not only possible, but easy to derive parametricity conditions in a dependently-typed language.

Further, it is possible to analyse parametricity properties of custom languages, via their embedding in a dependently-typed host language.

\section{Acknowledgments}

Thanks to Andreas Abel, Thierry Coquand, Peter Dybjer, Marc Lasson, Guilhem Moulin, Ulf Norell, Nicolas Pouillard and anonymous reviewers for providing us with very valuable feedback.

\section{References}

T. Altenkirch and C. McBride. Generic programming within dependently typed programming. In Proc. of the IFIP TC2/WG2.1 Working Conference on Generic Programming, pages 1-20. Kluwer, B.V., 2003.

H. P. Barendregt. Lambda calculi with types. Handbook of logic in computer science, 2:117-309, 1992.

M. Benke, P. Dybjer, and P. Jansson. Universes for generic programs and proofs in dependent type theory. Nordic J. of Computing, 10(4):265289, 2003.

J.-P. Bernardy. A proof of the abstraction theorem for pure type systems (unary case). http://www.cse.chalmers.se/ bernardy/ParDep/html/ Theorem.html, 2010.

J.-P. Bernardy, P. Jansson, and K. Claessen. Testing polymorphic properties. In Proc. of ESOP 2010, volume 6012 of LNCS. Springer, 2010a.

J.-P. Bernardy, P. Jansson, and R. Paterson. An abstraction theorem for pure type systems. Available from http://www.cse.chalmers.se/ bernardy/ ParDep/abstraction-pts.pdf, 2010b.

C. Böhm and A. Berarducci. Automatic synthesis of typed lambdaprograms on term algebras. Theor. Comp. Sci., 39(2-3):135-154, 1985.

T. Coquand. An analysis of Girard's paradox. In Proc. of LICS 1986, pages 227-236. IEEE Comp. Society Press, 1986.

T. Coquand. Pattern matching with dependent types. In Proc. of the Workshop on Types for Proofs and Programs, pages 66-79, 1992.

P. Dybjer. Inductive families. Formal Aspects of Computing, 6(4):440-465, 1994.

J. Gibbons and R. Paterson. Parametric datatype-genericity. In Proc. of WGP 2009, pages 85-93, Edinburgh, Scotland, 2009. ACM.

A. Gill, J. Launchbury, and S. L. Peyton Jones. A short cut to deforestation. In Proc. of FPCA, pages 223-232, Copenhagen, Denmark, 1993. ACM.

J. Y. Girard. Interprétation fonctionnelle et elimination des coupures de l'arithmétique d'ordre supérieur. Thèse d'état, Université de Paris 7, 1972.

R. Hinze. Church numerals, twice! J. Funct. Program., 15(1):1-13, 2005.

P. Johann. A generalization of short-cut fusion and its correctness proof. Higher-Order and Symbol. Comput., 15(4):273-300, 2002.

P. Johann and J. Voigtländer. The impact of seq on free theorems-based program transformations. Fundam. Inf., 69(1-2):63-102, 2005.

C. McBride and J. McKinna. The view from the left. J. Funct. Program., 14(01):69-111, 2004.

A. Miquel. Le Calcul des Constructions implicite: syntaxe et sémantique. Thèse de doctorat, Université Paris 7, 2001.

S. Monnier and D. Haguenauer. Singleton types here, singleton types there, singleton types everywhere. In Proc. of PLPV 2010, pages 1-8, Madrid, Spain, 2010. ACM.

G. Neis, D. Dreyer, and A. Rossberg. Non-parametric parametricity. In Proc. of ICFP 2009, pages 135-148, Edinburgh, Scotland, 2009. ACM.

$\mathrm{U}$. Norell. Towards a practical programming language based on dependent type theory. PhD thesis, Chalmers Tekniska Högskola, 2007.

N. Oury and W. Swierstra. The power of Pi. In Proc. of ICFP 2008, pages 39-50, Victoria, BC, Canada, 2008. ACM.

C. Paulin-Mohring. Inductive definitions in the system Coq - rules and properties. In Typed Lambda Calculi and Applications, pages 328-345. Springer, 1993.

G. Plotkin and M. Abadi. A logic for parametric polymorphism. In LNCS, volume 664, page 361-375. Springer-Verlag, 1993.

J. C. Reynolds. Types, abstraction and parametric polymorphism. Information processing, 83(1):513-523, 1983.

I. Takeuti. The theory of parametricity in lambda cube. Manuscript, 2004.

The Coq development team. The Coq proof assistant, 2010.

J. Voigtländer. Bidirectionalization for free! (Pearl). In Proc. of POPL 2009, pages 165-176, Savannah, GA, USA, 2009a. ACM.

J. Voigtländer. Free theorems involving type constructor classes: Funct. pearl. SIGPLAN Not., 44(9):173-184, $2009 \mathrm{~b}$. 


\begin{tabular}{|c|c|c|}
\hline & $\Gamma \vdash \mathrm{A}: \mathrm{B}$ & $\llbracket \Gamma \rrbracket \vdash \llbracket A \rrbracket: \llbracket \mathrm{B} \rrbracket \overline{\mathrm{A}}$ \\
\hline axiom & $\vdash s: s^{\prime}$ & $\vdash(\lambda \bar{x}: s \cdot \bar{x} \rightarrow \widetilde{s}): \bar{s} \rightarrow \widetilde{s^{\prime}}$ \\
\hline \multirow{2}{*}{ start } & $\Gamma \vdash \mathrm{A}: s$ & $\llbracket \Gamma \rrbracket \vdash \llbracket A \rrbracket: \overline{\mathrm{A}} \rightarrow \widetilde{s}$ \\
\hline & $\Gamma, x: \mathrm{A} \vdash x: \mathrm{A}$ & $\llbracket \Gamma \rrbracket, \overline{x: \mathrm{A}}, x_{R}: \llbracket \mathrm{A} \rrbracket \bar{x} \vdash x_{R}: \llbracket \mathrm{A} \rrbracket \bar{x}$ \\
\hline \multirow{2}{*}{ weakening } & $\begin{array}{l}\Gamma \vdash \mathrm{A}: \mathrm{B} \\
\Gamma \vdash \mathrm{C}: s \\
\end{array}$ & $\begin{array}{l}\llbracket \Gamma \rrbracket \vdash \llbracket A \rrbracket: \llbracket B \rrbracket \bar{A} \\
\llbracket \Gamma \rrbracket \vdash \llbracket C \rrbracket: \bar{C} \rightarrow \widetilde{s}\end{array}$ \\
\hline & $\Gamma, x: \mathrm{C} \vdash \mathrm{A}: \mathrm{B}$ & $\llbracket \Gamma \rrbracket, \overline{x: \mathrm{C}}, x_{R}: \llbracket \mathrm{C} \rrbracket \bar{x} \vdash \llbracket \mathrm{A} \rrbracket: \llbracket \mathrm{B} \rrbracket \overline{\mathrm{A}}$ \\
\hline \multirow{2}{*}{ product } & $\begin{array}{c}\Gamma \vdash \mathrm{A}: s_{1} \\
\Gamma, x: \mathrm{A} \vdash \mathrm{B}: s_{2}\end{array}$ & $\begin{array}{c}\llbracket \Gamma \rrbracket \vdash \llbracket \mathrm{A} \rrbracket: \overline{\mathrm{A}} \rightarrow \widetilde{s_{1}} \\
\llbracket \Gamma \rrbracket, \overline{x: \mathrm{A},}, x_{R}: \llbracket \mathrm{A} \rrbracket \bar{x} \vdash \llbracket \mathrm{B} \rrbracket: \overline{\mathrm{B}} \rightarrow \widetilde{s_{2}}\end{array}$ \\
\hline & $\Gamma \vdash(\forall x: \mathrm{A} . \mathrm{B}): s_{3}$ & $\overline{\llbracket \Gamma \rrbracket \vdash\left(\lambda \overline{f:(\forall x: \mathrm{A} \cdot \mathrm{B})} \cdot \forall \overline{x: \mathrm{A}} \cdot \forall x_{R}: \llbracket \mathrm{A} \rrbracket \bar{x} \cdot \llbracket \mathrm{B} \rrbracket(\overline{f x})\right):(\overline{\forall x: \mathrm{A} \cdot \mathrm{B}}) \rightarrow \widetilde{s_{3}}}$ \\
\hline \multirow{2}{*}{ application } & $\begin{array}{c}\Gamma \vdash \mathrm{F}:(\forall x: \mathrm{A} . \mathrm{B}) \\
\Gamma \vdash \mathrm{a}: \mathrm{A}\end{array}$ & $\begin{array}{c}\llbracket \Gamma \rrbracket \vdash \llbracket \mathrm{F} \rrbracket:\left(\forall \overline{x: \mathrm{A}} \cdot \forall x_{R}: \llbracket \mathrm{A} \rrbracket \bar{x} \cdot \llbracket \mathrm{B} \rrbracket(\overline{\mathrm{F} x})\right) \\
\llbracket \Gamma \rrbracket \vdash \llbracket \mathrm{a} \rrbracket: \llbracket \mathrm{A} \rrbracket \overline{\mathrm{a}} \\
\end{array}$ \\
\hline & $\Gamma \vdash \mathrm{Fa}: \mathrm{B}[x \mapsto \mathrm{a}]$ & $\llbracket \Gamma \rrbracket \vdash \llbracket \mathrm{F} \rrbracket \overline{\mathrm{a}} \llbracket \mathrm{a} \rrbracket: \llbracket \mathrm{B}[x \mapsto \mathrm{a}] \rrbracket(\overline{\mathrm{F}} \mathrm{a})$ \\
\hline \multirow{2}{*}{ abstraction } & $\begin{array}{c}\Gamma \vdash \mathrm{A}: s_{1} \\
\Gamma, x: \mathrm{A} \vdash \mathrm{B}: s_{2} \\
\Gamma, x: \mathrm{A} \vdash \mathrm{b}: \mathrm{B}\end{array}$ & 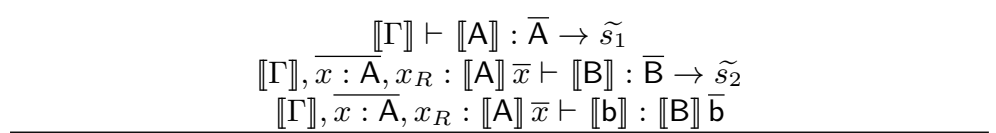 \\
\hline & $\Gamma \vdash(\lambda x:$ A. b $):(\forall x:$ A. B $)$ & $\llbracket \Gamma \rrbracket \vdash\left(\lambda \overline{x: \mathrm{A}} \cdot \lambda x_{R}: \llbracket \mathrm{A} \rrbracket \bar{x} \cdot \llbracket \mathrm{b} \rrbracket\right):\left(\forall \overline{x: \mathrm{A}} \cdot \forall x_{R}: \llbracket \mathrm{A} \rrbracket \bar{x} \cdot \llbracket \mathrm{B} \rrbracket \overline{\mathrm{b}}\right)$ \\
\hline \multirow{2}{*}{ conversion } & $\begin{array}{l}\Gamma \vdash \mathrm{A}: \mathrm{B} \\
\Gamma \vdash \mathrm{B}^{\prime}: s \\
\mathrm{~B}={ }_{\beta} \mathrm{B}^{\prime}\end{array}$ & $\begin{array}{c}\llbracket \Gamma \rrbracket \vdash \llbracket \mathrm{A} \rrbracket: \llbracket \mathrm{B} \rrbracket \overline{\mathrm{A}} \\
\llbracket \Gamma \rrbracket \vdash \llbracket \mathrm{B}^{\prime} \rrbracket: \overline{\mathrm{B}}^{\prime} \rightarrow \widetilde{s} \\
\llbracket \mathbb{B} \rrbracket=\beta \llbracket \mathrm{B}^{\prime} \rrbracket\end{array}$ \\
\hline & $\Gamma \vdash \mathrm{A}: \mathrm{B}^{\prime}$ & $\llbracket \Gamma \rrbracket \vdash \llbracket A \rrbracket: \llbracket \mathrm{B}^{\prime} \rrbracket \overline{\mathrm{A}}$ \\
\hline
\end{tabular}

Figure 6. Outline of a proof of Theorem 1 by induction over the derivation of $\Gamma \vdash A$ : B.

D. Vytiniotis and S. Weirich. Type-safe cast does no harm: Syntactic parametricity for $F \omega$ and beyond. Preliminary version of "Parametricity, Type Equality and Higher-order Polymorphism", 2009.

D. Vytiniotis and S. Weirich. Parametricity, type equality, and higher-order polymorphism. J. Funct. Program., 20(2):175-210, 2010.

P. Wadler. Theorems for free! In Proc. of FPCA 1989, pages 347-359, Imperial College, London, United Kingdom, 1989. ACM.

P. Wadler. The Girard-Reynolds isomorphism. Theor. Comp. Sci., 375(13):201-226, 2007.

P. Wadler and S. Blott. How to make ad-hoc polymorphism less ad hoc. In POPL'89, pages 60-76. ACM, 1989.

\section{A. Proof of the abstraction theorem}

In this appendix we sketch the proof of our main theorem, using the following lemma:

Lemma 4 (translation preserves $\beta$-reduction).

$$
\mathrm{A} \longrightarrow{ }_{\beta}^{*} \mathrm{~A}^{\prime} \Longrightarrow \llbracket \mathrm{A} \rrbracket \longrightarrow_{\beta}^{*} \llbracket \mathrm{A}^{\prime} \rrbracket
$$

Proof sketch. The proof proceeds by induction on the derivation of $A \longrightarrow{ }_{\beta}^{*} A^{\prime}$. The interesting case is where the term $A$ is a $\beta$ redex $(\lambda x: B$. C $)$ b. That case relies on the way $\llbracket-\rrbracket$ interacts with substitution:

$$
\llbracket \mathrm{b}[x \mapsto \mathrm{C}] \rrbracket=\llbracket \mathrm{b} \rrbracket[\bar{x} \mapsto \overline{\mathrm{C}}]\left[x_{R} \mapsto \llbracket \mathrm{C} \rrbracket\right]
$$

The remaining cases are congruences.

Theorem (abstraction). In a reflective PTS,

$$
\Gamma \vdash \mathrm{A}: \mathrm{B} \Longrightarrow \llbracket \Gamma \rrbracket \vdash \llbracket A \rrbracket: \llbracket B \rrbracket \bar{A}
$$

Proof sketch. A derivation of $\llbracket \Gamma \rrbracket \vdash \llbracket A \rrbracket: \llbracket B \rrbracket \bar{A}$ is constructed by induction on the derivation of $\Gamma \vdash A$ : $B$, using the syntactic properties of PTSs. We have one case for each typing rule: each type rule translates to a portion of a corresponding relational typing judgement, as shown in Figure 6.

For convenience, the proof uses a variant form of the abstraction rule; equivalence of the two systems follows from Barendregt [1992, Lemma 5.2.13]. The conversion case uses Lemma 4. 